\title{
1. NEOGENE CALCAREOUS NANNOFOSSIL BIOSTRATIGRAPHY AT SITES 812 THROUGH 818, NORTHEASTERN AUSTRALIAN MARGIN ${ }^{1}$
}

\author{
Stefan Gartner, ${ }^{2}$ Wuchang Wei, ${ }^{3}$ and Jih Ping Shyu ${ }^{2}$
}

\begin{abstract}
Two major transects were drilled across the northeastern Australian margin during Leg 133. The southern transect, consisting of Sites 812 through 818 (and 826), extends from the northern edge of Marion Plateau north to the southern edge of Queensland Plateau. Hemipelagic and pelagic sediments were recovered at all sites from the Holocene back to the upper Miocene sections. Calcareous nannofossils are a major constituent of these sediments. Nearly all of the low-latitude nannofossil biohorizons can be identified in the Pliocene and Pleistocene, which makes it possible to construct a reasonably accurate biochronology for each site. Sites 813 and 814 are similar in sedimentation rate and succession of biohorizons, and both have a hiatus (nondeposition) that straddles the Miocene/Pliocene boundary; much of the lower Pliocene is missing. Site 812 is also similar to the last two, but its biostratigraphic resolution is reduced because of poorer preservation in critical intervals. Sites 815 and 816 are broadly similar in that both have excellent biostratigraphic resolution in the Pleistocene and uppermost Pliocene intervals, even though the sedimentation rate for the two is different. Both sites have an expanded mid-Pliocene section. At Site 815 the expanded Pliocene section has been identified as a contourite; however, there is no evidence in the nannofossils for significant redeposition. Site 815 also has exceptionally good biostratigraphic resolution into the upper Miocene section. The ranges of Amaurolithus tricorniculatus and Amaurolithus amplificus were clearly identified at this site, although the lack of a magnetostratigraphy prevents assigning precise ages to them. Sites 817 and 818 also are similar, although Site 818 is more expanded. Both sites have a hiatus at the Pliocene/Pleistocene boundary.
\end{abstract}

\section{INTRODUCTION}

During Leg 133 the northeastern Australian margin was explored from just seaward of the Great Barrier Reef to the landward side of the Queensland Plateau (Fig. 1). Holes were drilled and cored along two transects, one an east-west transect across the Queensland Trough, roughly at the latitude of Cairns - the northern transect; the other a north-south transect across Townsville Trough, with the southern end of the transect at about the latitude of Townsville- the southern transect. This study is concerned with the southern transect, which includes Sites 812 through 818 (Fig. 2). Site 826 is a depth extension of Site 816, but as it yielded no nannofossils, it has not been considered further.

The sediments recovered at all sites of the southern transect are highly calcareous, approaching near $100 \%$ carbonate at some levels. The noncarbonate fraction-clays and other siliciclastics-is most abundant at the southern end of the transect (e.g., Site 815), which is just beyond the Great Barrier Reef. The carbonate fraction consists of detrital carbonate derived from nearby reefs and banks, and of the remains of pelagic organisms, prominent among them calcareous nannofossils and planktonic foraminifers.

The biostratigraphy of the holes drilled during Leg 133 is based principally on calcareous nannofossils, and this study is a detailed documentation of the biostratigraphy of Sites 812 through 818 . Abundant planktonic calcareous micro- and nannofossils were recovered from the sediment surface into the upper Miocene, beyond which pelagic micro- and nannofossils are extremely rare. This is true in some instances because deposition occurred in shallow water, in others because nannofossils may have been destroyed by diagenesis and lithification. Some nannofossils were found in these shallowwater carbonates and chalky limestones, but they are poorly preserved

\footnotetext{
'McKenzie, J.A., Davies, P.J., Palmer-Julson, A., et al., 1993. Proc. ODP, Sci. Results, 133: College Station, TX (Ocean Drilling Program). U.S.A.

2 Department of Oceanography, Texas A\&M University, College Station, TX 77843

${ }^{3}$ Scripps Institution of Oceanography, University of California at San Diego, La Jolla CA 92093, U.S.A.
}

and sporadic, and, consequently, a precise biochronology cannot be constructed from them.

Preservation of the calcareous nannofossils is highly variable. Rarely, nannofossils are pristine as at the water/sediment interface; more often, they are overgrown and fragmented.

\section{METHODS}

Nannofossil studies on board the JOIDES Resolution, as well as those done subsequently on land, were made on smear slides prepared from unprocessed sample material. All determinations were made with a light microscope. Total coccolith abundances were estimated and represent an impression of the proportion of the sediment that is nannofossils. This estimate, although useful, is inherently subjective and should be regarded with some caution. It is most valid as a relative measure for adjacent samples. Even so, the small sample used in the preparation of the smear slides may not be entirely representative of the bulk sediments at any level in a core. Abundances of individual species were recorded in six (semi-quantitative) categories that range from very rare (V) through very abundant (W) and have roughly the following numerical equivalents:

$$
\begin{aligned}
& \mathrm{V} \text { (very rare) }=<1 \text { specimen per } 50 \text { fields }(<0.04 \%) \\
& \mathrm{R} \text { (rare) }=1 \text { specimen per } 11-50 \text { fields }(\sim 0.04 \%-0.2 \%) \\
& \mathrm{F} \text { (few) }=1 \text { specimen per } 2-10 \text { fields }(\sim 0.2 \%-1 \%) \\
& \mathrm{C} \text { (common) }=1-5 \text { specimens per field }(\sim 1 \%-5 \%) \\
& \mathrm{A} \text { (abundant) }=6-25 \text { specimens per field }(\sim 5 \%-25 \%) \\
& \mathrm{W} \text { (very abundant) }=>25 \text { specimens per field }(>25 \%)
\end{aligned}
$$

This abundance ranking approximates a geometric progression where each category is roughly five times the preceding category.

All nannofossil biohorizons were determined to within $150 \mathrm{~cm}$, that is, to within one section, even where the checklist shows a larger sample spacing for the total assemblage determination (i.e., Hole $815 \mathrm{~A}$, Cores $13 \mathrm{H}$ to $48 \mathrm{X}$ ). Thus, the actual highest occurrence of a marker species may be anywhere within the $150-\mathrm{cm}$ interval above the designated highest occurrence; the actual lowest occurrence may be anywhere within $150 \mathrm{~cm}$ below the designated lowest occurrence. 


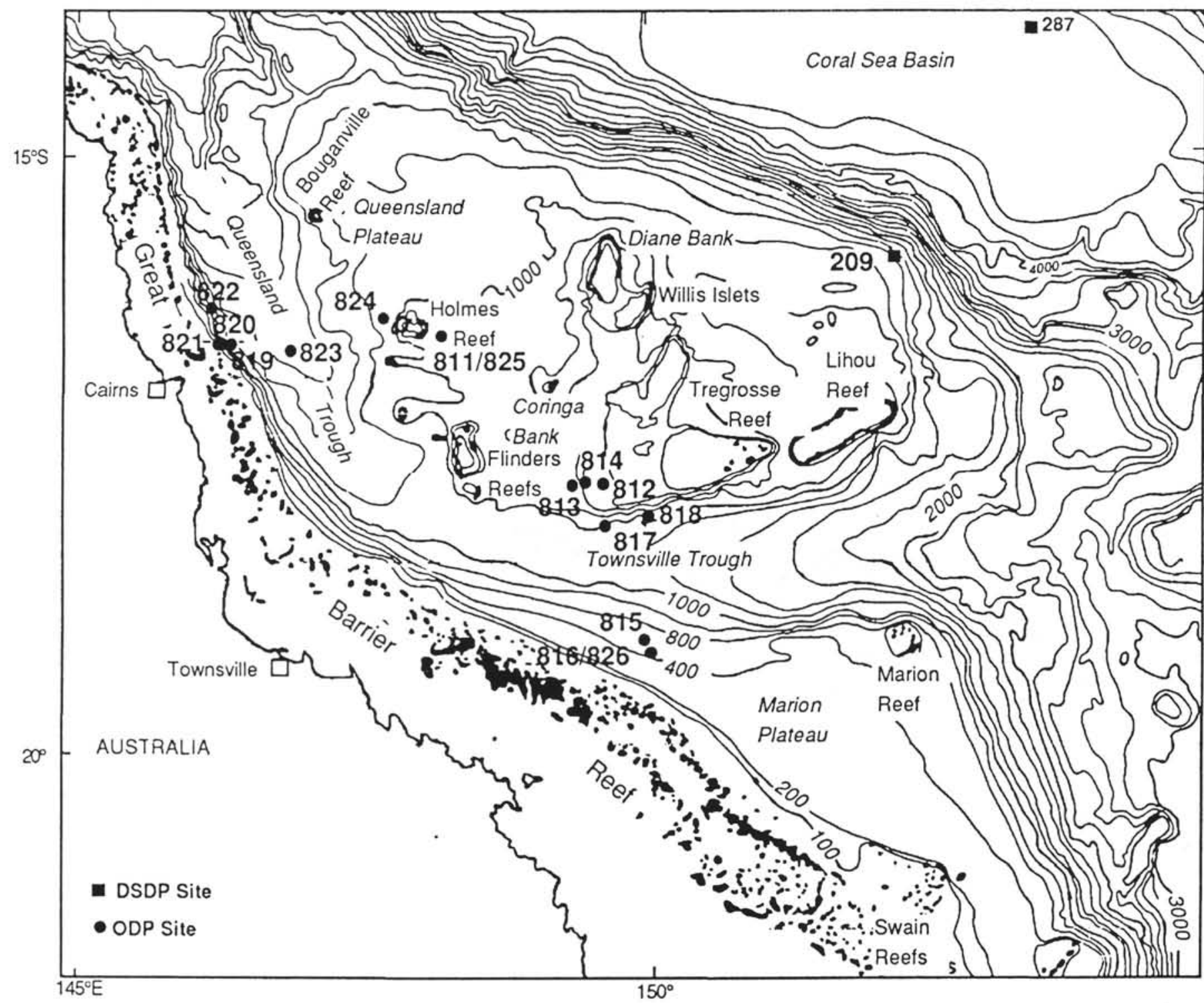

Figure 1. Location of Leg 133 sites on the northeastern Australian margin. Bathymetry in meters.

\section{DESCRIPTION OF FRAMEWORK}

The basic biostratigraphic framework for this study is the succession of calcareous nannofossil biohorizons given in Figure 3. The magnetic polarity time scale for the nannofossil biohorizons is from Berggren et al. (1985), with further revisions mainly from Baldauf et al. (1987). For reference purposes, both the nannofossil zonations of Okada and Bukry (1980) and of Martini (1971) are included. For the individual site overview, only the zonation of Okada and Bukry (1980) is given. Additional biohorizons have been included where sufficient documentation is available for their age or relative position. The biohorizons are inherently more accurate and precise, and, therefore, should be emphasized over zones and zonations. Among the added biohorizons are the lowest occurrence of Pseudoemiliania lacunosa and the lowest occurrence of Amaurolithus tricorniculatus, both in the lower Pliocene. Neither of these two can be considered an entirely dependable marker. The former species, although common, is mostly very small and altogether undistinguished at the lower limit of its occurrence; the latter is distinctive, but always rare or very rare. Nevertheless, the ages here assigned to these biohorizons are considered reliable and useful. In addition to the Leg 133 sites, these two biohorizons have been documented at the same relative position in Gulf of Mexico sediments (unpublished data).

The several upper Miocene biohorizons inserted in Figure 3 are from the North Atlantic Site 608 and are documented elsewhere (Gartner, 1992). The sites drilled during Leg 133 did not yield sufficient pelagic sections over this interval to materially test the utility of all of these biohorizons in the western Pacific Ocean, but they did prove useful for dating some sparsely fossiliferous intervals.

The Leg 133 sites did not yield a usable magnetostratigraphy, and the correlation of nannofossil biohorizons with the magnetic polarity time scale cannot be checked or improved with data from these sites. Moreover, the sedimentation rate at the Leg 133 sites is extremely variable, temporally and spatially, and any age estimates made by interpolation or extrapolation might have extremely large errors and, therefore, should be approached cautiously.

In the following site by site discussion, the biostratigraphy is presented primarily in terms of the depth $(\mathrm{mbsf}=$ meters below seafloor) of occurrence of relevant nannofossil biohorizons. Age assignments for each site are given graphically in the summary charts, along with nannofossil zonal assignments and the position of all identified nannofossil biohorizons. The ages for these biohorizons 


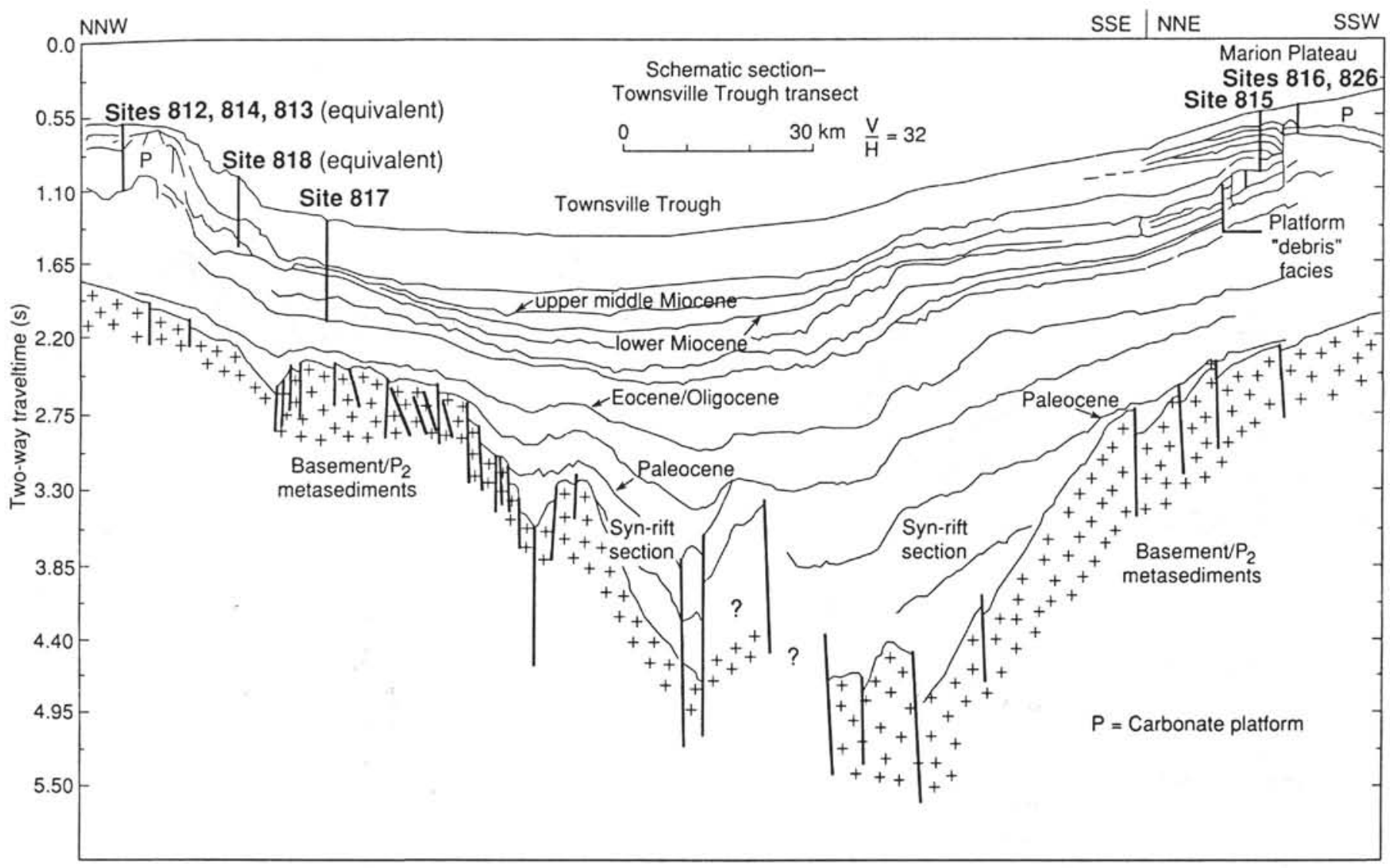

Figure 2. Schematic cross section of Townsville Trough, with location of Sites 812 through 818 (projected onto interpreted structural section).

(best current estimate) can be obtained from the chronological framework given in Figure 3. This format for the presentation was chosen to separate data and interpretation clearly so that future improvements to the chronology of nannofossil biohorizons can be readily applied to the data presented here.

\section{SITE REPORTS}

\section{Site 812}

Calcareous nannofossils are abundant in the uppermost $30 \mathrm{~m}$ of the section cored at this site and may constitute $10 \%$ to $20 \%$ of the sediments, which are almost entirely carbonate. Hole $812 \mathrm{~A}$ yielded poor recovery below about $20 \mathrm{~m}$ (Core $3 \mathrm{H}$ ), and the biostratigraphy of the site has been augmented with data from Hole 812C, in which recovery was reasonably good to about $115 \mathrm{~m}$ (Core 13H). The nannofossil biostratigraphy for Site 812 is summarized in Figure 4.

The youngest biohorizon recognized was the lowest occurrence of Emiliania huxleyi at $1.87 \mathrm{mbsf}(812 \mathrm{~A}-1 \mathrm{H}-2,37 \mathrm{~cm})$. That is followed by the highest occurrence of Pseudoemiliania lacunosa at 8.27 mbsf $(812 \mathrm{~A}-2 \mathrm{H}-3,37 \mathrm{~cm})$, and the top of the interval of dominance of small Gephyrocapsa at 17.77 mbsf (812A-3H-3, $37 \mathrm{~cm})$. This last species marks approximately the midpoint of the Pleistocene. Calcidiscus macintyre $i$ has its highest occurrence at $23.9 \mathrm{mbsf}$ in Hole $812 \mathrm{~A}(812 \mathrm{~A}-3 \mathrm{H}-\mathrm{CC})$ and at $24.4 \mathrm{mbsf}$ in Hole $812 \mathrm{C}(812 \mathrm{C}$ $36 \mathrm{X}-6,0 \mathrm{~cm})$. The slightly different depths of this datum in these two holes can be accounted for by sample spacing or incomplete recovery. The more complete core recovery in Hole $812 \mathrm{C}$ suggests that the 24.4-mbsf depth of the datum may be more nearly correct.

The highest occurrence of Discoaster brouweri was documented only in Hole $812 \mathrm{C}$, where it occurs at $25.9 \mathrm{mbsf}(812 \mathrm{C}-3 \mathrm{H}-7,0 \mathrm{~cm})$, and the highest occurrence of Discoaster pentaradiatus was documented in Hole $812 \mathrm{~A}$ at $27.9 \mathrm{mbsf}(812 \mathrm{~A}-4 \mathrm{H}-\mathrm{CC})$. Immediately below this level in both holes is the highest occurrence of Spheno- lithus abies, which in Hole $812 \mathrm{C}$ can be placed fairly precisely at $29.86 \mathrm{mbsf}(812 \mathrm{C}-5 \mathrm{H}-1,36 \mathrm{~cm})$.

The interval from the highest occurrence of Calcidiscus macintyrei to the highest occurrence of Sphenolithus abies clearly represents an attenuated section, while the lowermost part of the interval from the highest occurrence of Discoaster pentaradiatus to the highest occurrence of Sphenolithus abies almost certainly must contain a major hiatus that encompasses much of the late Pliocene interval. The poor core recovery in Core $4 \mathrm{H}$ of each of the two holes may be related to this hiatus and to the greatly attenuated section above it, because this interval of poor recovery is much too thin to account for the missing time interval.

The remaining section yielded far fewer nannofossils-from less than $1 \%$ to perhaps as much as $1 \%$ to $2 \%$-that consisted of a poorly preserved, low-diversity assemblage only marginally usable for biostratigraphy. Useful biohorizons are the highest occurrence of Reticulofenestra pseudoumbilicus at $58.36 \mathrm{mbsf}(812 \mathrm{C}-8 \mathrm{H}-1,36 \mathrm{~cm})$, which marks the early Pliocene to late Pliocene transition, and the highest occurrence of Discoaster quinqueramus at $86.36 \mathrm{mbsf}(812 \mathrm{C}-10-6 \mathrm{H}$, $36 \mathrm{~cm}$ ), which marks the Miocene/Pliocene boundary. Below this level no further datable nannofossil assemblages were recovered, and from Core 133-812C-12H downward, nannofossil yield approaches zero.

\section{Site 813}

Site 813 is located to the west of Site 812 and in slightly deeper water as well (see Fig. 1). The upper Neogene sediments recovered at this site have a strongly pelagic aspect, and calcareous nannofossils constitute from $20 \%$ to $80 \%$ of the sediments. Preservation is only moderate in the upper part of the section and deteriorates to poor below about 100 mbsf, which corresponds approximately to the Miocene/Pliocene boundary. Nevertheless, the biostratigraphy for the Pleistocene and upper Pliocene sections is good, with adequate biohorizons represented to permit reasonably accurate dating (Fig. 5). 


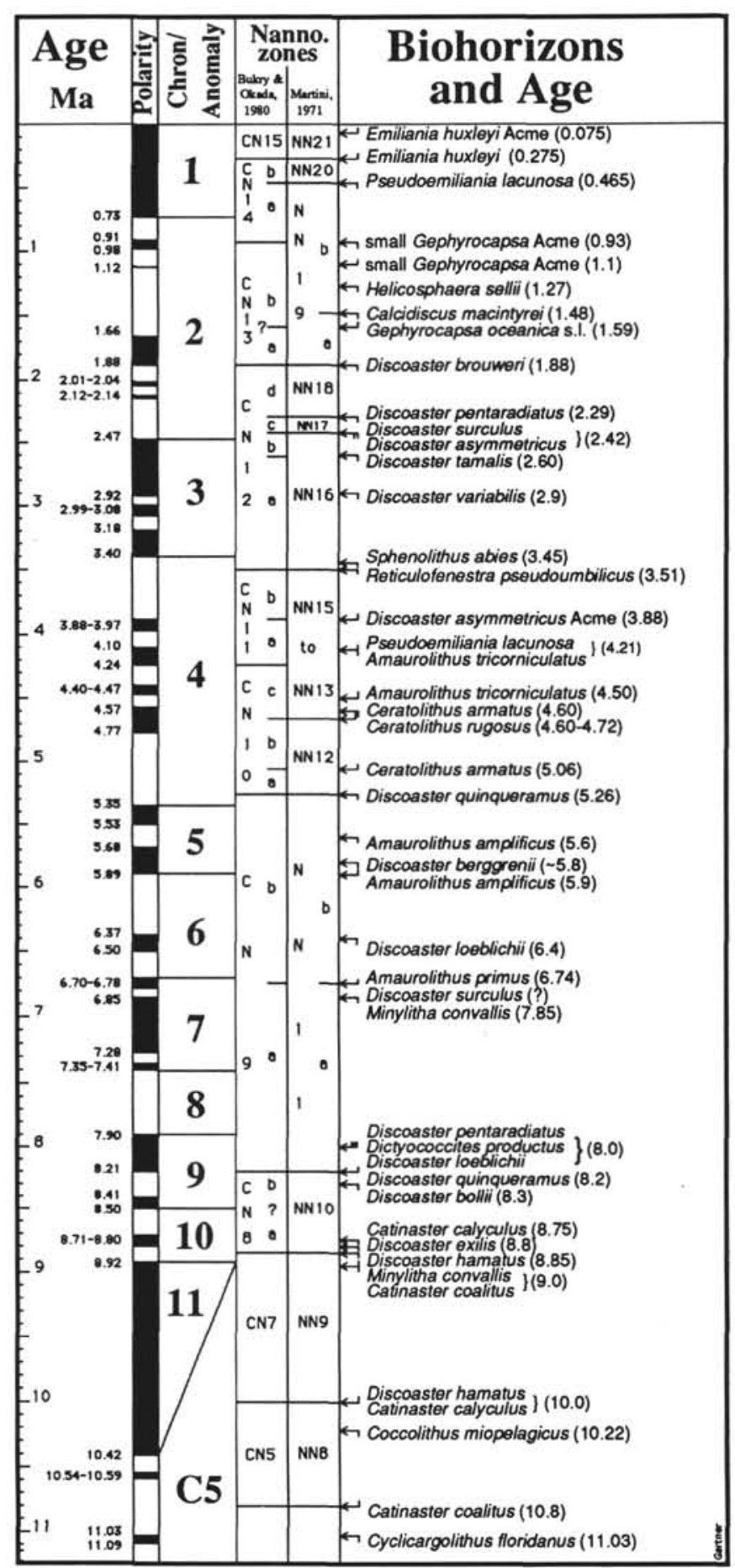

Figure 3. Biochronologic framework of calcareous nannofossil biohorizons and magnetostratigraphy compiled mainly from Berggren et al. (1985) and Baldauf et al. (1987).

The youngest biohorizon is the lowest occurrence of Emiliania huxleyi at $1.88 \mathrm{mbsf}(813 \mathrm{~A}-1 \mathrm{H}-2,38 \mathrm{~cm})$, followed by the highest occurrence of Pseudoemiliania lacunosa at $10.58 \mathrm{mbsf}(813 \mathrm{~A}-2 \mathrm{H}-4$, $38 \mathrm{~cm}$ ), and by the top of the small Gephyrocapsa dominance interval at $24.57 \mathrm{mbsf}(813 \mathrm{~A}-3 \mathrm{H}-7,38 \mathrm{~cm})$. This last datum has an age of about $0.93 \mathrm{Ma}$ and approximately marks the midpoint of the Pleistocene. The Helicosphaera sellii datum could not be identified at this site, and the next lower datum is the highest occurrence of Calcidiscus

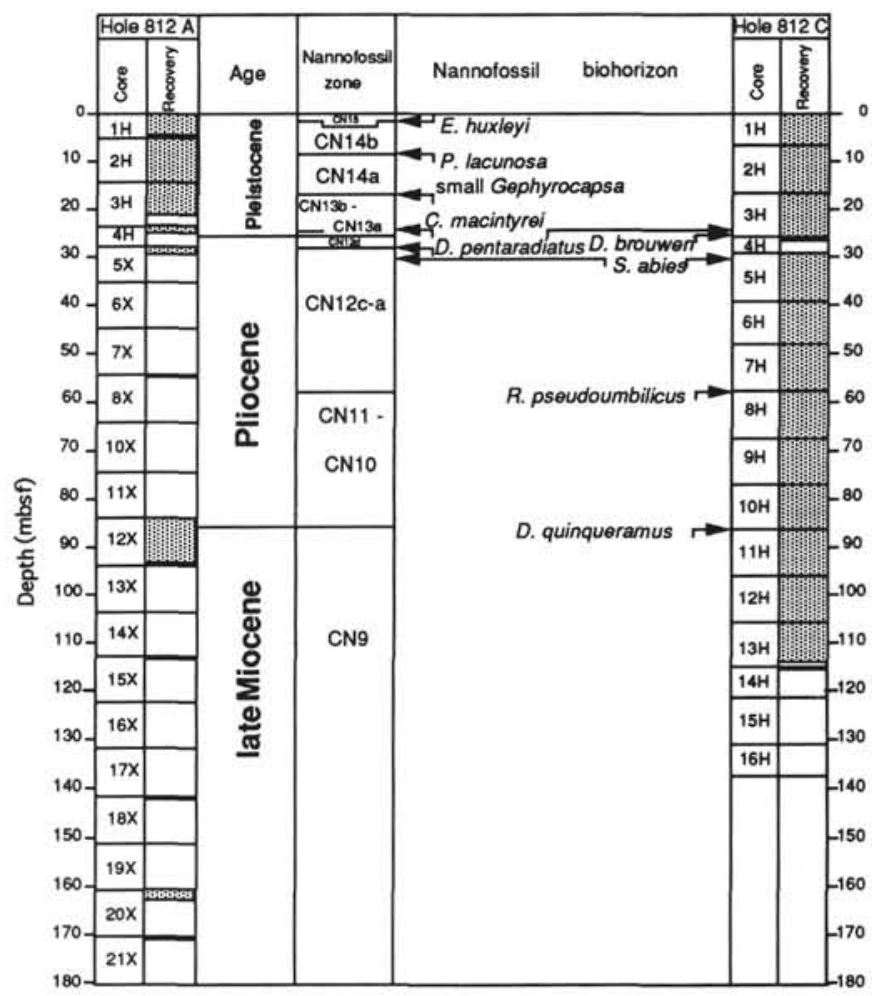

Figure 4. Summary of nannofossil biostratigraphy for Site 812 .

macintyrei at $34.07 \mathrm{mbsf}(813 \mathrm{~A}-4 \mathrm{H}-7,37 \mathrm{~cm})$, followed by the highest occurrence of Discoaster brouweri at 40.56 mbsf ( $813 \mathrm{~A}-5 \mathrm{H}-4,36$ $\mathrm{cm})$. This last species marks the Pliocene/Pleistocene boundary.

Upper Pliocene biohorizons are also well developed. The highest occurrences of Discoaster pentaradiatus, Discoaster surculus, and Discoaster tamalis are at $53.06 \mathrm{mbsf}(813 \mathrm{~A}-6 \mathrm{H}-6,36 \mathrm{~cm}), 54.5 \mathrm{mbsf}$ $(813 \mathrm{~A}-6 \mathrm{H}-7,36 \mathrm{~cm})$, and $58.06 \mathrm{mbsf}(813 \mathrm{~A}-7 \mathrm{H}-4,36 \mathrm{~cm})$, respectively. The midpoint of the Pliocene is marked by the highest occurrence of Reticulofenestra pseudoumbilicus at $81.56 \mathrm{mbsf}$ (813A-9H-6, $36 \mathrm{~cm}$ ), whereas the highest occurrence of Sphenolithus abies is just a short distance above at $80.06 \mathrm{mbsf}(813 \mathrm{~A}-9 \mathrm{H}-5,36 \mathrm{~cm})$. Although nannofossils remain abundant below this level, preservation deteriorates severely, and it is difficult to identify key markers, except for the highest occurrence of Discoaster quinqueramus at $100.56 \mathrm{mbsf}$ $(813 \mathrm{~A}-11 \mathrm{H}-6,36 \mathrm{~cm})$. The assemblage associated with Discoaster quinqueramus also contains forms very similar to the Pliocene Discoaster asymmetricus, which, however, may be found also associated with the much older Discoaster hamatus and Discoaster neohamatus. In any case, it is likely that a hiatus is present in the section at this level. Below the highest occurrence of Discoaster quinqueramus, the nannofossil assemblage, although abundant, consists mainly of generalized and poorly preserved reticulofenestrids and of heavily calcified, stemless sphenoliths and is not age-diagnostic.

\section{Site 814}

Site 814 lies near and slightly to the east of Site 813 . Its sediments are carbonate, much of which is coarse and displaced downslope as gravity-flow deposits. Calcareous nannofossils may be abundant (to $80 \%$ ) near the top of the section, but decrease sharply to $10 \%$ or less at about 100 mbsf and to less than $1 \%$ below about $100 \mathrm{mbsf}$. Between 0 and 100 mbsf, nannofossils may be extremely rare locally in coarse debris flows. Owing to the prevalence of displaced sediments, the biostratigraphy of this site has been revised significantly as a result of examination of additional samples. In spite of the large amount of 


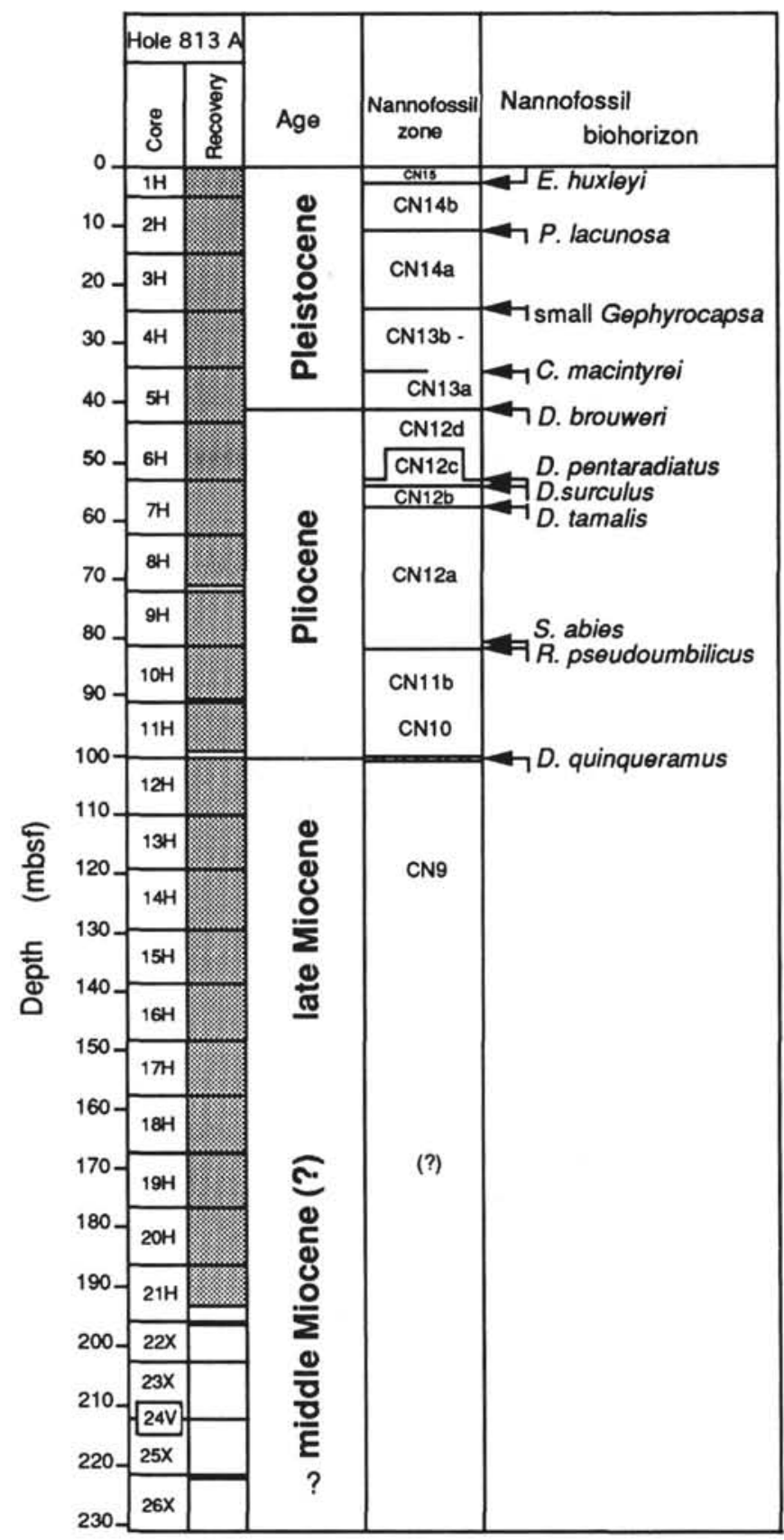

Figure 5. Summary of nannofossil biostratigraphy for Site 813.

redeposition, it is possible to establish a reasonably good biostratigraphy for the Pleistocene and the late Pliocene interval (i.e., to approximately $55 \mathrm{mbsf}$ ) (Fig. 6).

The youngest datum is the lowest occurrence of Emiliania huxleyi at $3.37 \mathrm{mbsf}(814 \mathrm{~A}-1 \mathrm{H}-2,37 \mathrm{~cm})$, which is followed by the highest occurrence of Pseudoemiliania lacunosa at 10.76 mbsf (814A-2H-3, $36 \mathrm{~cm}$ ), then by the top of the mid-Pleistocene interval of small Gephyrocapsa dominance at $21.76 \mathrm{mbsf}(814 \mathrm{~A}-3 \mathrm{H}-4,36 \mathrm{~cm})$. The lower Pleistocene highest occurrence of Calcidiscus macintyrei is at 29.76 mbsf $(814 \mathrm{~A}-4 \mathrm{H}-3,36 \mathrm{~cm})$, and the highest occurrence of Discoaster brouweri (i.e., the Pliocene/Pleistocene boundary) is at 34.26 mbsf ( $814 \mathrm{~A}-4 \mathrm{H}-6,36 \mathrm{~cm})$.

Three late Pliocene biohorizons also are well developed. The highest occurrence of Discoaster pentaradiatus is at $42.26 \mathrm{mbsf}$ $(814 \mathrm{~A}-5 \mathrm{H}-5,36 \mathrm{~cm})$, that of Discoaster surculus at $45.26 \mathrm{mbsf}(814 \mathrm{~A}-$
$5 \mathrm{H}-7,36 \mathrm{~cm})$, and of Discoaster tamalis at $53.26 \mathrm{mbsf}(814 \mathrm{~A}-6 \mathrm{H}-6$, $36 \mathrm{~cm}$ ). Poor core recovery from about 58 to $67 \mathrm{mbsf}$ renders this interval undecipherable. The highest occurrence of Sphenolithus abies is at $67.17 \mathrm{mbsf}$ ( $814 \mathrm{~A}-9 \mathrm{H}-1,67 \mathrm{~cm})$, although the near-contemporary highest occurrence of Reticulofenestra pseudoumbilicus cannot be identified in that same core. Nannofossils continue to be abundant in Cores $10 \mathrm{H}$ through $12 \mathrm{H}(10 \%-15 \%)$, but the assemblage is untypical and nearly devoid of discoasters. Rare, symmetrical, five-rayed discoasters (probably Discoaster quinqueramus) occur from $76.0 \mathrm{mbsf}$ $(814 \mathrm{~A}-10 \mathrm{H}-1,37 \mathrm{~cm})$ downward, and that level is taken as the Miocene/Pliocene boundary. Probably, a hiatus exists within Core $9 \mathrm{H}$.

Two additional markers were recorded from this site; however, both are problematic in that they are exceedingly rare and their preservation is only marginally adequate for identification. Calcidiscus premacintyrei occurs at 104.5 mbsf $(814 \mathrm{~A}-12 \mathrm{H}-\mathrm{CC})$, which suggests a middle Miocene age (>11.7 Ma) (Gartner, 1992); however, a five-rayed symmetrical discoaster was recorded from 110.85 mbsf (814A-13H-5, 36 $\mathrm{cm})$, which indicates a younger age $(<10.0 \mathrm{Ma})$ for this level. Clearly, the quality of the nannofossils is insufficient for determining reliable ages over this interval. The second biohorizon is the highest occurrence of Sphenolithus heteromorphus at $114.0 \mathrm{mbsf}(814 \mathrm{~A}-13 \mathrm{H}-\mathrm{CC})$ in a meager nannofossil assemblage. This species is found sporadically to 235 mbsf, but all of the specimens encountered are excessively overgrown and, at best, only suggest a middle Miocene age.

\section{Site 815}

Site 815 is located on the south flank of Townsville Trough, just beyond the northern edge of Marion Plateau in a thick pile of late Neogene-age hemipelagic to pelagic sediments (Fig. 1). Much of this pile of pelagic mud-some $400 \mathrm{~m}$ in all-is of mid-Pliocene age (nannofossil Subzone $\mathrm{CN} 12 \mathrm{a}$ and Zone $\mathrm{CN} 11$ ) and has been interpreted as a contourite deposited by bottom currents adjacent to the steep scarp of Marion Plateau (Fig. 2). Post-cruise study of the nannofossils from this site has led to a significant revision of the shipboard biostratigraphy, particularly with respect to the age of the interval from 40 to 160 mbsf. The biostratigraphy for Site 815 is summarized in Figure 7; a checklist for the site is given in Table 1.

The following important biohorizons were recognized for dating purposes at this site. The youngest biohorizon is the lowest occurrence of Emiliania huxleyi at $0.35 \mathrm{mbsf}(815 \mathrm{~A}-1 \mathrm{H}-1,35 \mathrm{~cm})$, indicating a very low latest Pleistocene and Holocene sedimentation rate. The highest occurrence of Pseudoemiliania lacunosa is next at $4.85 \mathrm{mbsf}$ $(815 \mathrm{~A}-1 \mathrm{H}-4,35 \mathrm{~cm})$, followed by the top of the interval of dominance of small Gephyrocapsa at 14.65 mbsf (815A-2H-5, $35 \mathrm{~cm}$ ), which here as elsewhere is marked by a radical reduction to near zero in the abundance of all Gephyrocapsa larger than $\sim 3 \mu \mathrm{m}$. At this site the base of this interval also can be identified and occurs at $16.12 \mathrm{mbsf}$ $(815 \mathrm{~A}-2 \mathrm{H}-7,32 \mathrm{~cm})$. The lower Pleistocene biohorizons include the highest occurrence of Helicosphaera sellii at $19.65 \mathrm{mbsf}$ (815A-3H$3,35 \mathrm{~cm}$ ) and the highest occurrence of Calcidiscus macintyrei at $21.15 \mathrm{mbsf}(815 \mathrm{~A}-3 \mathrm{H}-4,35 \mathrm{~cm})$. The Pliocene/Pleistocene boundary (as indicated by the highest occurrence of Discoaster brouweri) is at $25.65 \mathrm{mbsf}(815 \mathrm{~A}-3 \mathrm{H}-7,35 \mathrm{~cm})$, and below this were recognized three upper Pliocene discoaster biohorizons: the highest occurrence of Discoaster pentaradiatus at $32.15 \mathrm{mbsf}(815 \mathrm{~A}-4 \mathrm{H}-5,35 \mathrm{~cm})$; the highest occurrence of Discoaster surculus at $37.15 \mathrm{mbsf}(815 \mathrm{~A}-5 \mathrm{H}-2$, $35 \mathrm{~cm}$ ); and the highest occurrence of Discoaster tamalis at 43.15 mbsf (815A-5H-6, $35 \mathrm{~cm})$.

Below this, sedimentation rate increases nearly twenty-fold, (from about $7 \mathrm{~m} / \mathrm{m}$.y. to about $130 \mathrm{~m} / \mathrm{m}$.y.), and the next lower biohorizon, the highest occurrence of Sphenolithus abies, is encountered at 117.66 mbsf (815A-13H-6, $36 \mathrm{~cm})$. The highest occurrence of Reticulofenestra pseudoumbilicus is at $158.9 \mathrm{mbsf}(815 \mathrm{~A}-18 \mathrm{X}-1,21 \mathrm{~cm})$, which marks approximately the mid-point of the Pliocene. These last two biohorizons are here interpreted differently than was done in the Initial Reports volume (Davies, McKenzie, Palmer-Julson, et al., 1991), 


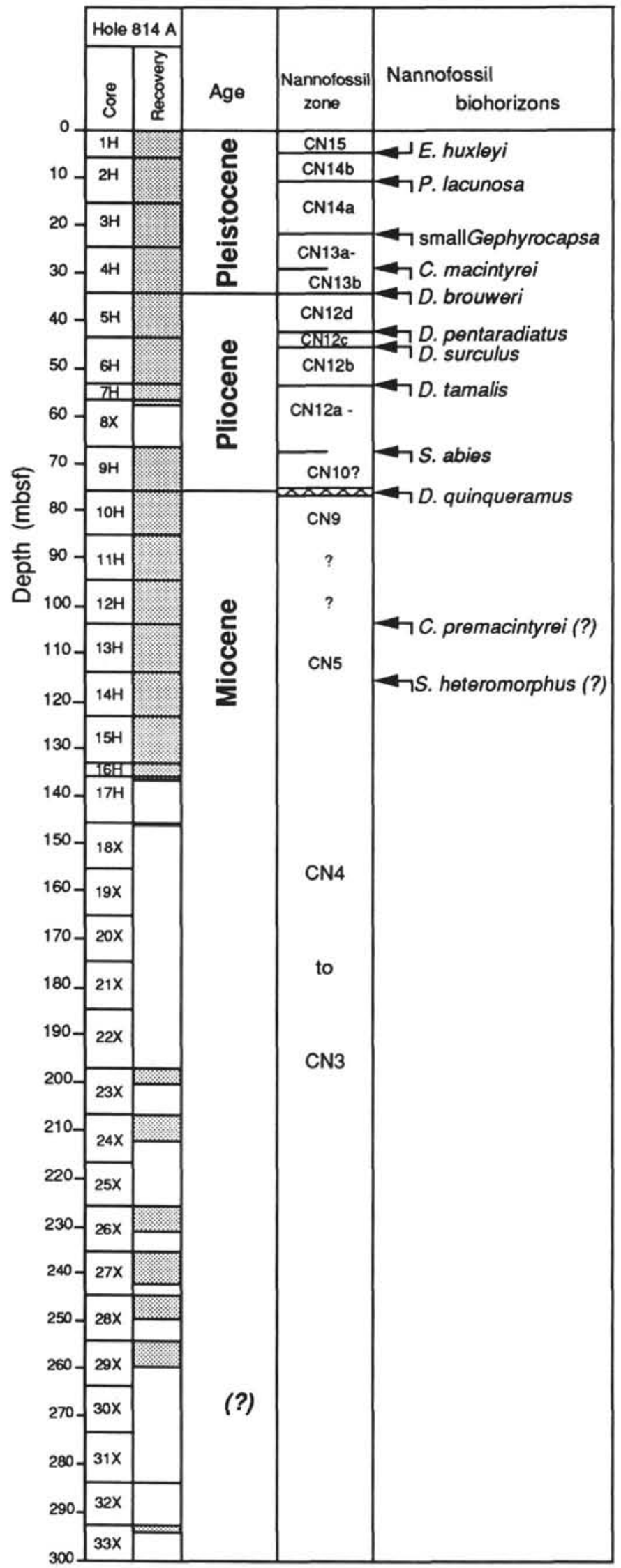

Figure 6. Summary of nannofossil biostratigraphy for Site 814 . because both may appear sparsely and scattered in samples above the levels identified here. Such occurrences, however, almost certainly represent redeposition. Comparable high sedimentation rates also prevailed during the latter part of the early Pliocene, as the next biohorizon, the highest occurrence of Amaurolithus tricorniculatus, is at $309.60 \mathrm{mbsf}$ (815A-34X-CC), which is also close to the lowest occurrence of Pseudoemiliania lacunosa at $309.26 \mathrm{mbsf}(815 \mathrm{~A}-34 \mathrm{X}-7,36 \mathrm{~cm})$.

From this level downward, sediments again accumulated at a reduced rate-about $38 \mathrm{~m} / \mathrm{m}$.y. - to the Miocene/Pliocene boundary. The next two lower biohorizons are not cited generally; these are the highest occurrence of Ceratolithus armatus at $327.10 \mathrm{mbsf}$ (815A$36 \mathrm{X}-6,30 \mathrm{~cm})$ and the lowest occurrence of Amaurolithus tricorniculatus at $330.86 \mathrm{mbsf}(815 \mathrm{~A}-37 \mathrm{X}-2,36 \mathrm{~cm})$. Both seem to be useful markers for the early Pliocene, but both will require further documentation in additional sections. The lowest occurrence of Ceratolithus rugosus is at $339.07 \mathrm{mbsf}(815 \mathrm{~A}-38 \mathrm{X}-1,37 \mathrm{~cm})$, and lowest occurrences of birefringent ceratoliths (Ceratolithus acutus or Ceratolithus armatus $)$ are at $350.25 \mathrm{mbsf}(815 \mathrm{~A}-39 \mathrm{X}-2,35 \mathrm{~cm})$.

The Miocene/Pliocene boundary is placed at the highest occurrence of Discoaster quinqueramus at 353.25 mbsf ( $815 \mathrm{~A}-39 \mathrm{X}-4,35$ $\mathrm{cm})$, and the closely related Discoaster berggrenii has its highest occurrence at $377.79 \mathrm{mbsf}(815 \mathrm{~A}-42 \mathrm{X}-1,39 \mathrm{~cm})$. The highest and lowest occurrences of Amaurolithus amplificus are also within this core at $379.27 \mathrm{mbsf}(815 \mathrm{~A}-42 \mathrm{X}-2,37 \mathrm{~cm})$ and $386.77 \mathrm{mbsf}(815 \mathrm{~A}-$ $42 X-7,37 \mathrm{~cm}$ ), respectively. Nannofossils continue in abundance through Core $45 \mathrm{X}$, and the nonbirefringent ceratoliths Amaurolithus primus and Amaurolithus delicatus are present to the bottom of this core at $415.70 \mathrm{mbsf}(815 \mathrm{~A}-45 \mathrm{X}-\mathrm{CC})$. Below this point, nannofossils become exceedingly rare; symmetrical discoasters bearing five rays continue to the bottom of Core $47 \mathrm{X}$ at $434.90 \mathrm{mbsf}$ (815A-47X-CC), indicting a late Miocene to possibly latest middle Miocene (maximum) age for this level.

\section{Site $\mathbf{8 1 6}$}

Site 816 is located slightly south of Site 815 on Marion Plateau, where a relatively thin pelagic section covers the shallow-water carbonate core of the plateau. The pelagic to hemipelagic section extends to approximately $90 \mathrm{mbsf}$ (through Core $10 \mathrm{H}$ ), and calcareous nannofossils are a significant constituent of the sediment over this interval. Most of this $90 \mathrm{~m}$ of hemipelagic sediment is of mid-Pliocene age, and the latest Pliocene and Pleistocene records are relatively attenuated, though apparently complete, within the upper $15 \mathrm{~m}$ of the section. Below approximately $90 \mathrm{mbsf}$, the limestones are barren of calcareous nannofossils. A biostratigraphic summary for Site 816 is given in Figure 8.

The youngest recognizable biohorizon is the lowest occurrence of Emiliania huxleyi at $0.37 \mathrm{mbsf}(816 \mathrm{~A}-1 \mathrm{H}-1,37 \mathrm{~cm})$, followed in the very next lower sample by the highest occurrence of Pseudoemiliania lacunosa at $1.87 \mathrm{mbsf}(816 \mathrm{~A}-1 \mathrm{H}-2,37 \mathrm{~cm})$. The top of the interval of small Gephyrocapsa dominance can be recognized at $4.87 \mathrm{mbsf}$ $(816 \mathrm{~A}-1 \mathrm{H}-4,37 \mathrm{~cm})$, although the base of that interval as well as the highest occurrence of Helicosphaera sellii could not be identified. The next lower biohorizon is the highest occurrence of Calcidiscus macintyrei at $10.37 \mathrm{mbsf}(816 \mathrm{~A}-2 \mathrm{H}-3,37 \mathrm{~cm})$, and the Pliocene/Pleistocene boundary was placed at $13.37 \mathrm{mbsf}(816 \mathrm{~A}-2 \mathrm{H}-5,37 \mathrm{~cm})$, at the highest occurrence of Discoaster brouweri. The three uppermost Pliocene discoaster biohorizons follow in a short interval of sediments. The highest occurrence of Discoaster pentaradiatus is seen at $15.37 \mathrm{mbsf}$ (816A-3H-1, $37 \mathrm{~cm})$; the highest oceurrence of Discoaster surculus is at $18.37 \mathrm{mbsf}(816 \mathrm{~A}-3 \mathrm{H}-3,37 \mathrm{~cm})$; and the highest occurrence of Discoaster tamalis is at $19.87 \mathrm{mbsf}(816 \mathrm{~A}-3 \mathrm{H}-4,37 \mathrm{~cm})$.

Below this point, the sedimentation rate increases markedly (from about $7 \mathrm{~m} / \mathrm{m}$.y. to about $67 \mathrm{~m} / \mathrm{m} . \mathrm{y}$.), and the next biohorizon, the highest occurrence of Sphenolithus abies, is at $43.5 \mathrm{mbsf}$ (816A-5H-CC), followed substantially farther down by the only slightly older Reticulofenestra pseudoumbilicus at $76.86 \mathrm{mbsf}(816 \mathrm{~A}-9 \mathrm{H}-4,36 \mathrm{~cm})$. This 


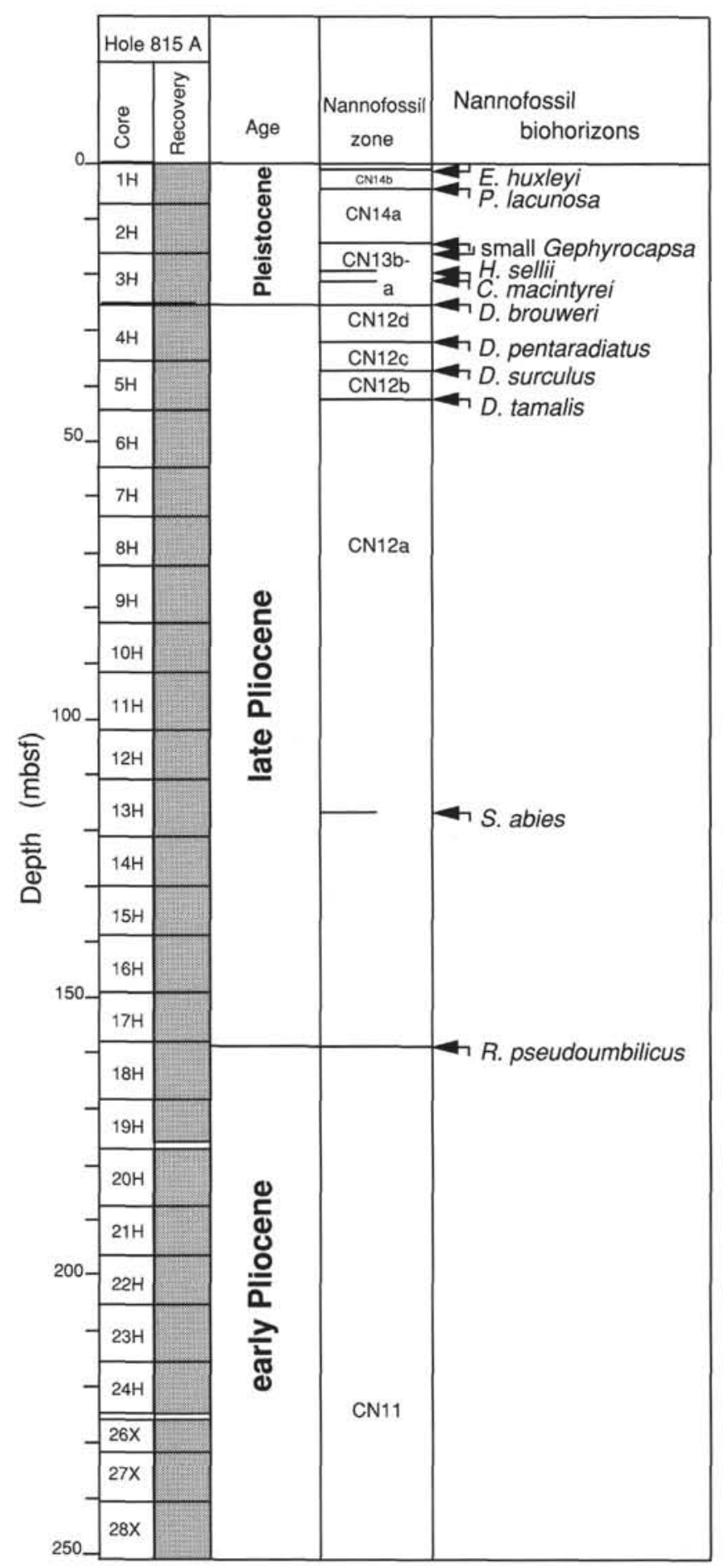

Figure 7. Summary of nannofossil biostratigraphy for Site 815.

datum marks the midpoint of the Pliocene and is the oldest biohorizon recognized at this site. Presumably, the transition from a shallowwater carbonate environment to a pelagic-hemipelagic setting above occurred just before the extinction of Reticulofenestra pseudoumbilicus, whose age has been determined as $3.51 \mathrm{Ma}$ (see Fig. 3).

\section{Site 817}

Site 817 was drilled below the northern flank of Townsville Trough and just below the southern edge of Queensland Plateau. The

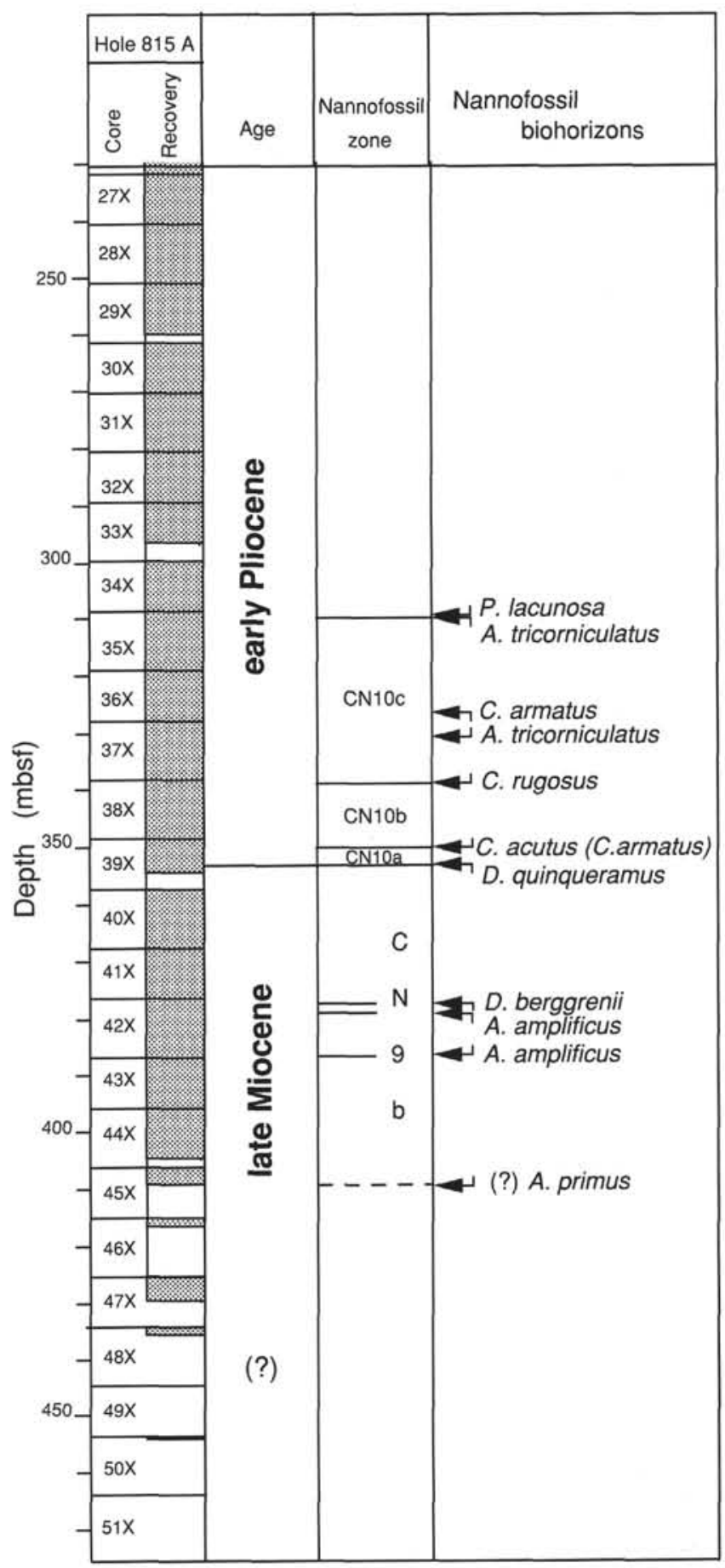

sediment is mostly carbonate, chiefly periplatform ooze, but with an abundant pelagic component. Calcareous nannofossils are generally abundant and may constitute from a few percent to more than $50 \%$ (estimated) of the bulk of the sediment. Abundance and diversity of nannofossils decrease dramatically at about $200 \mathrm{mbsf}$, and consequently, the biostratigraphy of the section below that level is less certain than that above it. The Pleistocene and late Pliocene-age intervals contain large amounts of sediment derived from the nearby carbonate platforms and thus are relatively more expanded than the early Pliocene- and late Miocene-age intervals. The biostratigraphy 
Table 1. Calcareous nannofossils from Hole 815A.

\begin{tabular}{|c|c|c|c|c|c|c|c|c|c|c|c|c|c|c|c|c|c|c|c|c|c|c|c|c|c|c|c|}
\hline & 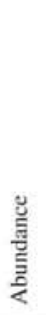 & & 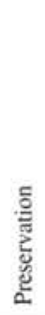 & & 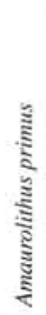 & 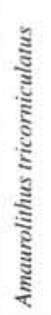 & 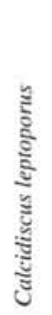 & 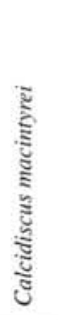 & 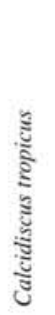 & 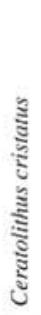 & 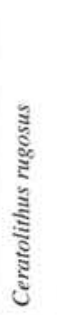 & 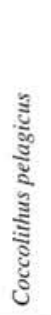 & 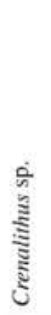 & 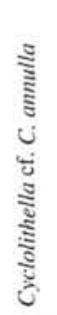 & 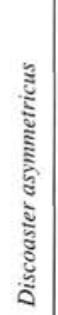 & 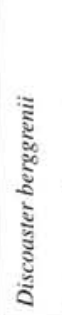 & 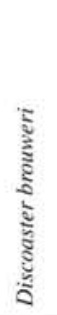 & 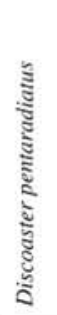 & 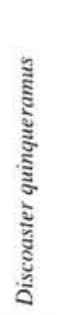 & 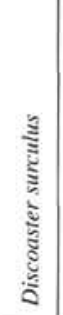 & 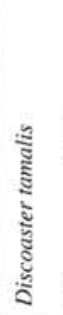 & 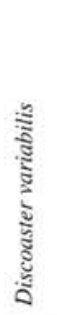 & 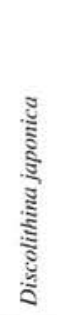 & 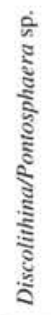 & 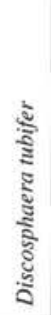 & 莺 & 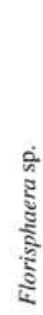 \\
\hline $1-1.35$ & A & & M & . & & , & C & & & & . & . & . & . & . & & & . & & . & . & . & . & . & C & W & A \\
\hline 35 & w & & P & . & & . & F & . & & & . & & . & . & . & & . & . & & . & & & & . & . & 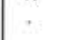 & : \\
\hline $1-3.35$ & W & & P & & & . & V & & & . & . & & . & . & . & & & & & . & & . & . & & V & . & C \\
\hline $1-4,35$ & A & & P & & & & $\mathrm{R}$ & . & . & & . & & & . & . & . & . & & & . & . & . & & . & v & . & V \\
\hline $1-5,35$ & C & & P & & & & $\mathrm{R}$ & . & . & & & & . & . & . & . & . & . & & . & . & . & & & . & . & . \\
\hline $2-1.35$ & A & . & P & & & . & $\mathrm{R}$ & & & & & & & & . & & . & . & & . & . & . & . & . & V & . & . \\
\hline 35 & A & & P & & & & R & & . & & . & & & & . & & . & . & & . & . & . & & & F & . & . \\
\hline $2-3.35$ & W & & M & & & & F & . & . & & & & . & . & . & . & & & & . & . & . & & $\mathrm{R}$ & v & . & . \\
\hline $2-4,35$ & W & . & $P$ & & & & F & & & & & & & & . & & & . & & . & . & . & . & $\mathrm{v}$ & . & . & . \\
\hline $2-5,35$ & A & . & P & . & . & . & R & . & & . & . & . & & . & . & & & . & . & . & . & & & v & . & . & . \\
\hline $2-6,32$ & W & . & P & & . & . & $\mathrm{R}$ & & & & & & & . & . & & & . & & . & . & & . & . & v & & . \\
\hline & w & . & P & . & . & . & F & . & & & & . & . & . & . & & . & . & . & . & . & & & v & . & & . \\
\hline $3-1,35$ & W & & G & . & . & . & $\mathrm{F}$ & & & . & . & . & . & . & . & . & & & . & . & . & . & . & V & v & . & $\mathrm{R}$ \\
\hline $3-2,35$ & W & & G & . & . & & F & & & & 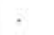 & . & . & & . & & & . & - & . & . & & v & v & V & . & R \\
\hline $3-3,35$ & A & . & M & . & . & & $\mathrm{F}$ & & . & . & & . & . & & . & & . & . & . & . & . & , & . & v & . & , & $\mathrm{R}$ \\
\hline $3-4,35$ & A & . & M & . & . & & F & F & . & . & & . & . & . & . & . & . & . & . & . & . & . & . & $\mathrm{R}$ & $\mathrm{F}$ & . & \\
\hline 5,35 & A & & M & . & . & . & $\mathrm{R}$ & $\mathrm{R}$ & & & . & . & . & . & . & & . & . & . & . & . & & . & & F & . & $\mathbf{R}$ \\
\hline 5,35 & A & . & M & . & . & . & & $\mathrm{R}$ & . & & - & . & & & . & & . & . & . & . & . & . & . & . & . & . & $\mathbf{R}$ \\
\hline $3-7,35$ & A & . & P & . & . & & $\mathrm{F}$ & & . & & . & $\mathrm{R}$ & & & . & & V & . & & . & & . & & . & . & . & $\mathrm{F}$ \\
\hline $4-1,15$ & A & & P & & . & & v & & . & . & . & V & & & . & & & & & . & & . & . & . & . & . & $\mathrm{F}$ \\
\hline $4-2,35$ & c & . & P & . & . & & $\mathrm{F}$ & v & . & & . & . & A & . & . & & v & . & . & . & & . & & $\mathrm{v}$ & . & & $\mathrm{F}$ \\
\hline 4 & c & & P & . & & & $\mathrm{F}$ & $\mathrm{R}$ & . & & . & $\mathrm{R}$ & A & v & . & & : & . & & . & . & . & & v & $\mathrm{v}$ & . & \\
\hline $4-4.35$ & c & & P & . & . & & v & $\mathrm{R}$ & . & & . & $\mathrm{R}$ & A & & . & & $\mathrm{F}$ & & & . & & . & & $\mathrm{R}$ & . & . & \\
\hline $4-5.35$ & A & & P & & & & $\mathrm{F}$ & $\mathrm{F}$ & . & . & . & $\mathrm{R}$ & A & $\mathrm{R}$ & . & . & F & V & & . & & . & . & $\mathrm{F}$ & & . & . \\
\hline $4-6.35$ & A & & $P$ & & & & R & $\mathrm{V}=$ & . & . & & . & A & $:$ & . & & $R$ & $R$ & & . & & . & . & R & v & . & . \\
\hline $5-1,35$ & w & & M & & & & $R$ & & . & & & $\mathrm{R}$ & $\mathrm{F}$ & $\mathrm{F}$ & . & & F & $\mathrm{R}$ & & . & & . & . & $\mathrm{R}$ & V & . & . \\
\hline 35 & A & & P & & & & $\mathrm{R}$ & $\mathrm{R}$ & & & 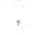 & s & : & & . & & F & & & $\mathrm{R}$ & & . & . & $\mathrm{R}$ & : & . & . \\
\hline $5-3,35$ & A & . & M & & . & & $\mathrm{F}$ & C & & & & V & C & $\mathrm{R}$ & . & . & C & & . & V & & & v & $\mathrm{R}$ & V & . & . \\
\hline $5-4,35$ & u & . & M & . & . & . & $\mathrm{F}$ & $R$ & & . & v & V & . & $\mathrm{F}$ & . & & $F$ & 2 & . & . & . & & & v & . & . & . \\
\hline $5-5,35$ & W & . & M & . & . & . & $\mathrm{F}$ & $\mathrm{F}$ & . & & & $\mathrm{F}$ & . & $\mathrm{R}$ & . & . & $\mathrm{F}$ & $\mathrm{F}$ & . & . & . & . & & $\mathrm{R}$ & . & . & . \\
\hline $5-6,35$ & w & . & G & . & . & . & C & C & & & $\mathrm{v}$ & v & . & & $\mathrm{v}$ & . & $\mathrm{F}$ & $\mathrm{F}$ & . & V & & & V & $\mathrm{R}$ & $\mathrm{R}$ & & F \\
\hline 35 & w & . & G & . & . & & . & $\mathrm{F}$ & . & & F & $\mathrm{F}$ & . & $\mathrm{F}$ & & & & & . & $\mathrm{F}$ & & . & & $\mathrm{F}$ & v & . & V \\
\hline 6. & u & . & G & . & . & . & C & V & . & & . & $\therefore$ & . & $\mathrm{R}$ & R & . & $\mathrm{F}$ & C & . & $\mathrm{R}$ & & . & v & $\mathrm{R}$ & . & . & \\
\hline $6-2,35$ & w & . & G & . & . & & $\mathrm{R}$ & $\mathrm{F}$ & . & & 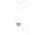 & v & & $\vec{F}$ & R & & $\mathrm{F}$ & & . & $\mathrm{v}$ & $\mathrm{v}$ & v & & $\mathrm{F}$ & $\mathrm{v}$ & . & \\
\hline $6-3,35$ & A & . & M & . & . & & $\mathrm{F}$ & v & . & . & . & $\mathrm{R}$ & & & . & . & $\mathrm{F}$ & $\mathrm{F}$ & . & $\mathrm{R}$ & v & $\mathrm{R}$ & $\mathrm{R}$ & F & & . & . \\
\hline $6-4,35$ & w & . & M & . & . & & $\mathrm{F}$ & $\mathrm{R}$ & . & . & & $\mathrm{F}$ & . & 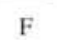 & . & & $F$ & F & . & $R$ & & V & v & $\mathrm{F}$ & . & . & . \\
\hline & & & $\mathrm{N}$ & . & . & & $\mathrm{F}$ & R & . & & $v$ & & & & $\therefore$ & & & & . & & & . & I & F & & . & \\
\hline & u & . & M & . & . & & $\mathrm{F}$ & $\mathrm{F}$ & . & . & V & $\mathrm{F}$ & & & & & & & . & $\mathrm{R}$ & & & $\therefore$ & $\mathrm{R}$ & & . & . \\
\hline 73 & u & & P & & & & $\mathrm{F}$ & $*$ & . & & & 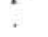 & . & $\mathrm{F}$ & v & $\cdot$ & $\mathrm{R}$ & F & . & & & . & & $\mathrm{R}$ & & . & . \\
\hline $7-1,35$ & w & & P & & . & . & $\mathrm{F}$ & v & & . & & R & . & C & . & . & & $\mathrm{R}$ & . & V & & . & . & $\mathrm{R}$ & . & . & . \\
\hline 23 & u & & $P$ & . & . & & F & v & . & & & & . & & v & & & & & v & & & . & $\mathrm{R}$ & . & & . \\
\hline & A & & P & & . & & $\mathrm{R}$ & $\mathrm{R}$ & & & & v & . & & & & & & & & & & & . & . & . & \\
\hline $7-4,35$ & u & & P & & & & $\mathrm{F}$ & . & & & & $\mathrm{R}$ & . & R & V & & V & $\mathrm{R}$ & . & V & & & $R$ & . & . & . & . \\
\hline & A & & P & & . & & $\mathrm{F}$ & . & & & & . & . & R & $\therefore$ & & v & 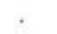 & . & V & & & V & R &. & . & \\
\hline $7-6,35$ & W & . & P & . & . & . & $\mathrm{R}$ & $R$ & & & & & . & C & . & . & $\mathrm{R}$ & $R$ & . & $\mathrm{R}$ & . & & . & R & . & . & . \\
\hline & u & . & P & & & & $\mathrm{R}$ & & & & & v & & & . & & & . & & V & & & . & $R$ & . & . & . \\
\hline & u & . & $\mathrm{P}$ & & & & $\mathrm{F}$ & $\mathrm{R}$ & & $x^{\prime}$ & . & & & C & & & & & & & V & & V & $\mathrm{R}$ & . & . & . \\
\hline & A & . & $\mathrm{P}$ & . & . & & $\mathrm{R}$ & & . & & & . & . & & . & & $\mathrm{v}$ & V & . & V & . & , & $\mathrm{R}$ & . & & & \\
\hline & A & . & P & . & . & & F & v & . & . & 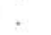 & . & & F & . & & V & V & . & & & . & . & $\mathrm{R}$ & & & . \\
\hline $8-4,35$ & A & . & P & . & . & . & $\mathrm{F}$ & F & . & . & . & . & & $\mathrm{F}$ & . & . & & R & . & $\mathrm{R}$ & & $\mathrm{R}$ & . & R & . & . & \\
\hline & u & . & $\mathrm{P}$ & & . & & $\mathrm{F}$ & $\mathrm{F}$ & . & 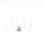 & & . & & & . & & & & . & V & & . & V & $\mathrm{R}$ & . & & . \\
\hline & u & & M & & . & & $\mathrm{R}$ & $\mathrm{F}$ & . & & . & . & & & . & . & V & & & V & & . & . & F & & & . \\
\hline & u & . & $\mathrm{P}$ & & . & & $\mathrm{F}$ & $\mathrm{F}$ & . & . & & . & A & c & . & $=$ & & R & . & V & & & v & $\mathrm{R}$ & . & & . \\
\hline $9-1,3$ & A & & M & & & $\sigma^{\circ}$ & $\mathrm{F}$ & $\mathrm{R}$ & . & ${ }^{\circ}$ & 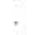 & V & A & $\mathrm{F}$ & . & & & $\mathrm{R}$ & . & V & V & & . & $\mathrm{v}$ & V & & \\
\hline $9-2,37$ & W & & M & & . & . & $\mathrm{F}$ & $\mathrm{F}$ & & . & & . & A & $\mathrm{F}$ & . & & . & $\ddot{v}$ & . & $\mathrm{R}$ & & & . & v & $\therefore$ & & \\
\hline $9-3,37$ & W & & M & . & . & . & $\mathrm{F}$ & F & . & & . & . & C & $\mathrm{F}$ & V & . & $\mathrm{R}$ & $\mathrm{R}$ & & V & . & & . & $\mathrm{V}$ & . & . & \\
\hline
\end{tabular}

Note: Key to symbols: $\cdot=$ Not present; $\mathrm{V}=$ Very rare; $\mathrm{R}=$ Rare; $\mathrm{F}=\mathrm{Few} ; \mathrm{C}=$ Common; $\mathrm{A}=\mathrm{Abundant} ; \mathrm{W}=$ Very abundant. 
Table 1 (continued).

\begin{tabular}{|c|c|c|c|c|c|c|c|c|c|c|c|c|c|c|c|c|c|c|c|c|c|c|c|c|c|c|c|}
\hline & 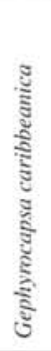 & 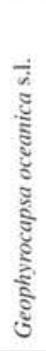 & 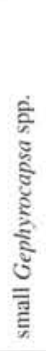 & 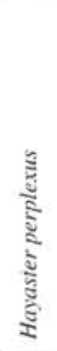 & 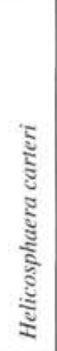 & 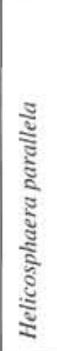 & 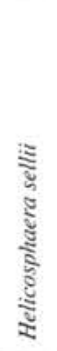 & 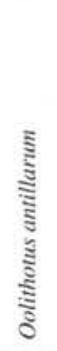 & 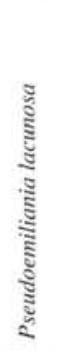 & 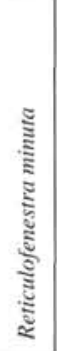 & 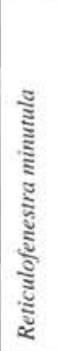 & 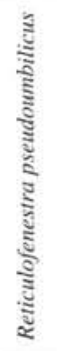 & 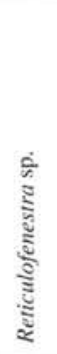 & 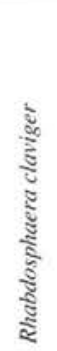 & 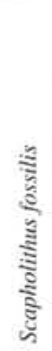 & 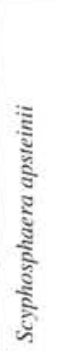 & 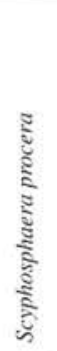 & 咅 & 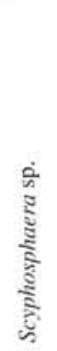 & 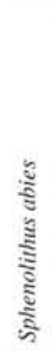 & 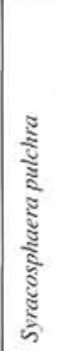 & 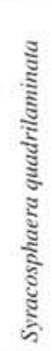 & 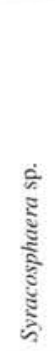 & 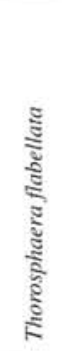 & 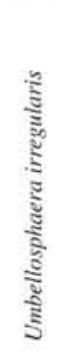 & 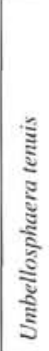 & 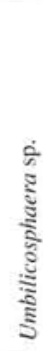 \\
\hline $1-1,35$ & $\mathrm{~F}$ & A & w & & $\mathrm{F}$ & . & & v & . & . & . & . & $\mathrm{R}$ & v & $\mathrm{R}$ & & & & . & . & $\mathrm{F}$ & & $\mathrm{R}$ & C & v & $\mathrm{v}$ & C \\
\hline 1. & w & . & W & & v & . & & . & & . & & . & . & $\mathrm{F}$ & . & & & & & . & v & & & $\mathrm{R}$ & $\therefore$ & $v$ & $\mathrm{~F}$ \\
\hline & w & $\mathrm{F}$ & $\mathrm{F}$ & & $\mathrm{R}$ & . & & & $\mathrm{R}$ & . & . & . & & & & & & & & & $\mathrm{R}$ & & & V & V & & $\mathrm{F}$ \\
\hline $1-4.35$ & w & $\mathrm{F}$ & . & & $\mathrm{R}$ & . & & & $\mathrm{R}$ & . & . & & & v & $\mathrm{v}$ & & . & . & . & & v & & V & $\mathrm{R}$ & V & . & C \\
\hline $1-5.35$ & C & $\mathrm{R}$ & $\mathrm{R}$ & . & . & . & . & & $\mathrm{F}$ & . & . & . & . & : & & . & . & . & & & v & & . & & . & & $\mathrm{F}$ \\
\hline 1,35 & C & F & $\mathrm{R}$ & & . & . & & & $\mathrm{F}$ & . & . & & & & $\mathrm{V}$ & & . & & & & $\mathrm{R}$ & & $\mathrm{R}$ & . & . & & $\mathrm{F}$ \\
\hline & C & C & A & & V & . & & & $\mathrm{F}$ & . & . & & & & V & & & & & & & & & & & & $\mathrm{F}$ \\
\hline 3,35 & w & $\mathrm{R}$ & A & . & $\mathrm{F}$ & . & & . & C & . & . & . & & $\mathrm{R}$ & . & . & . & . & & . & $\mathrm{R}$ & & & & & & C \\
\hline .35 & A & V & C & . & . & . & . & . & C & . & & & $\mathrm{R}$ & & & & . & . & & . & $\mathrm{F}$ & V & & v & & & C \\
\hline $2-5,35$ & . & R & A & . & $\mathrm{F}$ & . & & & C & . & & & & v & v & & . & . & & . & $\mathrm{R}$ & & . & & & & $\mathrm{F}$ \\
\hline $2-6,32$ & & & W & . & . & . & & . & A & . & . & . & . & $\mathrm{R}$ & v & . & . & . & & & $\mathrm{R}$ & . & & & . & . & $\mathrm{F}$ \\
\hline & $\mathrm{R}$ & $\mathrm{R}$ & u & . & $\mathrm{F}$ & . & & & A & . & & & . & & V & & & & & & F & . & & & v & & C \\
\hline .35 & A & $\mathrm{F}$ & W & . & C & . & . & V & A & . & & & & $\mathrm{F}$ & $\mathrm{R}$ & . & . & . & & . & C & V & v & & . & & $\mathrm{F}$ \\
\hline & C & F & W & . & V & . & . & $\therefore$ & C & . & . & & & $\mathrm{R}$ & $\mathrm{R}$ & & & & . & & $\mathrm{F}$ & V & . & & & & $\mathrm{F}$ \\
\hline $3-3,35$ & C & & A & & $\mathrm{F}$ & . & $\mathrm{v}$ & V & C & . & & & & $\hat{R}$ & $\mathrm{R}$ & & . & . & . & . & $\mathrm{F}$ & . & . & . & & . & $\mathrm{F}$ \\
\hline $3-4.35$ & & & A & . & $\mathrm{F}$ & . & & . & C & . & & & & $\mathrm{R}$ & V & $\mathrm{v}$ & . & . & & . & $\mathrm{F}$ & & & & & & $\mathrm{F}$ \\
\hline & F & & A & . & $\mathrm{F}$ & . & . & & c & . & . & & & i & R & & . & . & & & $\mathrm{F}$ & . & . & & & & $\mathrm{F}$ \\
\hline & & $\mathrm{R}$ & A & . & $\mathrm{F}$ & . & v & . & A & . & & & & & $\mathrm{R}$ & & 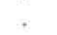 & . & & . & $\mathrm{F}$ & . & . & . & . & & F \\
\hline $3-7.35$ & & & C & v & $\mathrm{F}$ & . & V & $\mathrm{v}$ & A & . & . & & & $\mathrm{F}$ & $\mathrm{R}$ & & . & . & . & . & $\mathrm{F}$ & . & . & . & . & & $\mathrm{R}$ \\
\hline $4-1,15$ & v & v & A & & $\mathrm{F}$ & . & . & . & C & . & . & & & $\mathrm{F}$ & . & & . & . & . & & $\mathrm{F}$ & . & & . & & & . \\
\hline & & . & & . & $\mathrm{R}$ & . & V & & C & . & & & & F & v & & . & . & . & & & 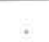 & & & & & \\
\hline & & & C & . & $\mathrm{F}$ & . & V & . & ( & , & & & & & V & & . & . & . & 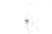 & 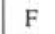 & & . & & & & \\
\hline & & & C & & F & . & $\mathrm{R}$ & . & $c$ & . & . & & & F & . & & . & . & V & . & $F$ & . & . & . & & & \\
\hline 4 & & & & & $\mathrm{~F}$ & . & $\mathrm{R}$ & . & A & . & & & & F & $\mathrm{F}$ & V & . & . & & & C & . & & . & . & & . \\
\hline $4-6,35$ & . & & F & & C & . & F & . & A & . & . & & & F & $\mathrm{R}$ & & . & . & . & . & $\mathrm{F}$ & . & & . & . & & . \\
\hline 5 & . & . & . & & C & . & $\mathrm{R}$ & . & A & A & C & & & C & $\mathrm{F}$ & & $\mathrm{R}$ & . & . & & & . & . & . & . & & . \\
\hline & & & & & $\mathrm{F}$ & . & R & . & $A$ & 1 & & . & . & & $\mathrm{R}$ & & & & $\mathrm{v}$ & & & . & . & . & & & . \\
\hline & & & & & C & . & . & v & A & c & & & & i & & $\mathrm{R}$ & v & V & : & . & & . & & . & & & . \\
\hline & & & $\mathrm{R}$ & & C & . & . & : & A & c & & & & c & $\mathrm{R}$ & & & & . & . & C & & . & & & & . \\
\hline $5-5,35$ & . & . & R & & C & . & . & . & A & A & A & & & C & $\mathrm{R}$ & $\mathrm{R}$ & . & . & . & . & C & . & & & . & & . \\
\hline & & & . & & C & . & $\mathrm{R}$ & v & C & A & A & . & & C & V & & & & . & . & & . & . & . & & & . \\
\hline & & & & & $\mathrm{r}$ & & . & . & ( & & & & & & & & & & & & & & & & & & . \\
\hline & & & & & $\mathrm{F}$ & . & . & . & $A$ & A & & & & s & v & & 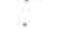 & & & & & & & . & & & . \\
\hline & & & F & & F & . & . & . & F & A & & & & $\mathrm{F}$ & V & & . & . & & & & & & & & & \\
\hline $6-3,35$ & . & & $\mathrm{F}$ & & $\mathrm{F}$ & . & . & , & $\mathrm{F}$ & A & A & . & & & & . & , & V & & & F & & . & & . & & \\
\hline & & . & C & & C & . & & & A & A & & ${ }^{\circ}$ & & & $\mathrm{R}$ & & & & & & & & & . & & & . \\
\hline & . & . & . & & & & . & v & & & & . & & & & & & & & & & . & & & & & \\
\hline & & . & w & & c & . & . & . & A & $\mathrm{F}$ & & & & 1 & v & & & . & $\therefore$ & & C & & & & & & \\
\hline & & & w & & $\mathrm{F}$ & . & V & . & A & $\mathrm{F}$ & & & & 1 & V & & v & . & & & & & & & & & \\
\hline $7-1,35$ & & . & A & . & $\mathrm{R}$ & . & & & A & . & A & . & & $\mathrm{R}$ & & & & . & & & F & & . & & . & & \\
\hline & & & A & . & $\mathrm{F}$ & & & & $A$ & A & & . & & & $\mathrm{R}$ & & & v & & & & & & & & & . \\
\hline & & & A & . & i & 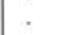 & & & & & & . & & & & & $\mathrm{V}$ & & & & & & & . & & & \\
\hline & & & u & & c & . & V & & , & A & & $\therefore$ & & , & & & & & & & & & & . & & . & . \\
\hline & & & A & & C & . & . & & A & . & , & & & F & & v & . & & . & & & & & . & & & . \\
\hline $7-6,35$ & & & A & & C & . & & & C & A & A & . & & $\mathrm{R}$ & & & . & & & & $\mathrm{F}$ & & & . & . & & \\
\hline & & . & $A$ & & $\mathrm{~F}$ & & & & & & & . & & & & & & & & & & & & & . & & \\
\hline & & . & A & & $\mathrm{r}$ & & & & & & & . & . & i & $\mathrm{R}$ & & & . & & . & & . & . & & . & & \\
\hline & & & A & & $\mathrm{F}$ & & & & 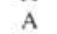 & & & & . & & 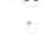 & & & & & & & & & & & . & . \\
\hline & . & . & A & & $\mathrm{R}$ & . & & & A & A & 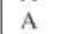 & & & $\mathrm{F}$ & . & & & & & . & & . & . & & . & & \\
\hline $8 \cdot 4,35$ & . & . & C & . & $\mathrm{R}$ & . & & & C & A & A & & & V & & & . & v & . & . & $\mathrm{F}$ & . & . & & . & & \\
\hline & . & . & A & . & $\mathrm{F}$ & & & . & ( & . & & & & & V & & & v & & & & & . & & . & & \\
\hline & . & . & A & & $\mathrm{F}$ & & & . & C & . & & & & $\mathrm{F}$ & & . & . & & . & . & & . & . & & . & 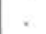 & \\
\hline & . & . & C & . & F & . & & . & $\mathrm{F}$ & A & 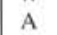 & & & & $\mathrm{v}$ & . & v & & . & & & . & . & & . & . & . \\
\hline & . & . & . & . & $\mathrm{R}$ & . & $\mathrm{v}$ & & $\mathrm{F}$ & A & 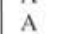 & . & & $\mathrm{R}$ & v & . & & & & & $F$ & . & . & & . & . & \\
\hline $9-2,37$ & . & & $\mathrm{F}$ & & $\mathrm{F}$ & $\theta^{\circ}$ & $x^{\circ}$ & & $\mathrm{F}$ & A & A & & & R & v & & v & v & . & & $\mathrm{F}$ & . & . & & . & . & . \\
\hline $9 \cdot 3.37$ & . & . & A & & $\mathrm{F}$ & . & V & & $\mathrm{F}$ & A & A & & & $F$ & V & $\mathrm{v}$ & & & & . & $\mathrm{F}$ & . & . & & . & & . \\
\hline
\end{tabular}


Table 1 (continued).

\begin{tabular}{|c|c|c|c|c|c|c|c|c|c|c|c|c|c|c|c|c|c|c|c|c|c|c|c|c|c|c|c|}
\hline & $\begin{array}{l}\text { पू. } \\
\text { हूँ } \\
\text { है }\end{array}$ & & 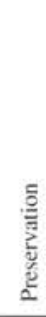 & & 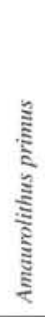 & 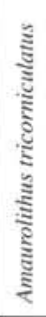 & 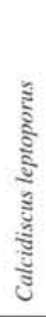 & 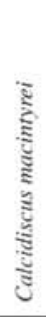 & 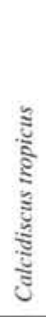 & 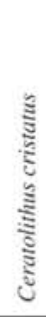 & 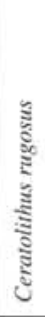 & 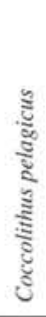 & 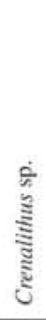 & 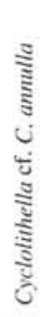 & 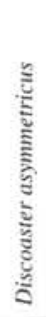 & 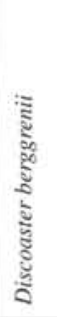 & 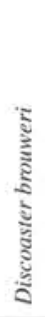 & 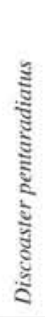 & 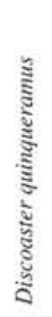 & 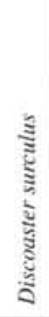 & 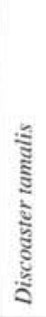 & 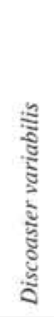 & 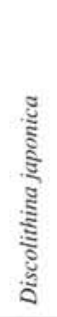 & 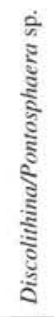 & 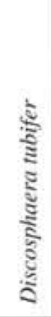 & 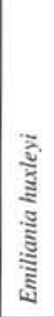 & 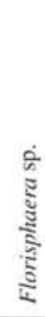 \\
\hline $9-4,37$ & W & & M & . & . & . & $\mathrm{F}$ & . & . & . & . & . & $\mathrm{F}$ & $\mathrm{F}$ & . & . & $\mathrm{R}$ & $\mathrm{R}$ & . & V & & & V & $\mathrm{R}$ & V & & \\
\hline $9-5,37$ & W & & M & & & & C & V & & & & v & C & C & . & . & $\mathrm{R}$ & $\mathrm{R}$ & & V & v & . & V & $\mathrm{R}$ & & . & \\
\hline $9-6,37$ & W & & M & & . & . & $\mathrm{F}$ & $\mathrm{F}$ & . & . & & . & A & $\mathrm{F}$ & . & . & $\mathrm{R}$ & $\mathrm{R}$ & . & & v & . & . & $\mathrm{R}$ & . & . & \\
\hline $9-7,37$ & A & & P & & & . & $\mathrm{R}$ & R & . & . & . & . & $\because$ & C & . & . & . & . & . & v & . & . & . & . & . & & . \\
\hline $10-1,36$ & W & . & M & . & . & . & $\mathrm{F}$ & $\mathrm{F}$ & . & . & . & V & C & $\mathrm{F}$ & V & . & v & . & . & . & v & . & & v & . & & \\
\hline $10-2,36$ & A & & P & . & $\therefore$ & . & $\mathrm{F}$ & $\mathrm{R}$ & . & . & . & . & . & $\mathrm{F}$ & v & . & v & $\mathrm{R}$ & & v & . & . & . & . & . & & . \\
\hline $10-3,36$ & A & & P & & . & . & $\mathrm{F}$ & $\mathrm{R}$ & . & $\therefore$ & . & . & . & $\mathrm{R}$ & V & & v & $\mathrm{R}$ & & v & & & . & v & & & \\
\hline $10-4,36$ & A & & P & & & . & $\mathrm{R}$ & $\mathrm{R}$ & & & & & C & $\mathrm{R}$ & . & . & V & . & . & $\mathrm{R}$ & & . & . & v & . & . & \\
\hline $10-5,36$ & A & & $\mathrm{P}$ & & . & . & $\mathrm{R}$ & $\mathrm{F}$ & . & . & & . & . & $\mathrm{F}$ & . & . & v & & & . & & & & $\mathrm{R}$ & . & . & \\
\hline $10-6,36$ & A & . & $P$ & . & . & . & $\mathrm{R}$ & F & . & . & . & V & . & $\mathrm{R}$ & . & . & v & v & . & & & . & & & v & & \\
\hline $10-7,36$ & A & & P & . & . & . & $\mathrm{R}$ & $\mathrm{F}$ & . & & . & . & . & $\mathrm{R}$ & . & . & . & . & . & v & & . & . & & & . & \\
\hline $11-1,36$ & A & & P & & . & . & & $\mathrm{R}$ & & & & & & & . & . & . & . & . & $\mathrm{R}$ & & . & . & & . & . & . \\
\hline $11-2,36$ & A & & P & & & . & $\mathrm{F}$ & $\mathrm{F}$ & & & & & . & $\mathrm{R}$ & . & & v & v & . & v & & & . & v & . & . & \\
\hline $11-3,36$ & A & . & P & & & & $\mathrm{R}$ & F & & & & & A & $\mathrm{F}$ & . & . & v & . & . & V & & . & . & $\mathrm{R}$ & & & \\
\hline $11-4,36$ & A & . & P & . & & & $\mathrm{R}$ & $\mathrm{F}$ & & & & & & C & & . & $\mathrm{R}$ & v & . & & & . & V & $\mathrm{F}$ & & & . \\
\hline $11-5,36$ & A & . & P & . & & & R & $\mathrm{F}$ & & & & & & $\mathrm{F}$ & & . & & & . & $\mathrm{R}$ & & . & . & $\mathrm{R}$ & & . & . \\
\hline $11-6,36$ & A & & P & . & & & $\mathrm{R}$ & $\mathrm{F}$ & & & & & A & $\mathrm{F}$ & & & V & $\mathrm{R}$ & . & . & & . & . & $\mathrm{R}$ & . & . & . \\
\hline $11-7.36$ & W & & $\mathrm{P}$ & . & & & $\mathrm{F}$ & $\mathrm{F}$ & & & & & & F & & & v & R & & $\mathrm{R}$ & & & & $\mathrm{R}$ & . & . & . \\
\hline $12-1,36$ & w & & $P$ & & . & , & F & F & & . & , & . & . & F & v & & & $\mathrm{R}$ & . & & & V & & $\mathrm{R}$ & & & \\
\hline $12-2,36$ & W & & P & & . & . & $\mathrm{R}$ & $\mathrm{F}$ & & & & . & . & $\mathrm{F}$ & & . & $\mathrm{R}$ & C & & . & . & . & . & $\mathrm{R}$ & v & . & . \\
\hline $13-1,36$ & A & & P & & & & $\mathrm{F}$ & $\mathrm{F}$ & & & & & . & $\mathrm{F}$ & & & . & & & & & & & . & . & & \\
\hline $14-1.36$ & A & & $P$ & . & & . & $\mathrm{F}$ & v & & & . & & . & $\mathrm{F}$ & . & . & $\mathrm{R}$ & & . & $\mathrm{R}$ & & & & $\mathrm{R}$ & & & \\
\hline $15-1,36$ & A & & $P$ & . & . & . & $\mathrm{F}$ & $\mathrm{R}$ & . & . & & . & & $\mathrm{F}$ & . & . & & & & & & . & . & $\mathrm{R}$ & $\mathrm{v}$ & & . \\
\hline $16-1,36$ & A & & P & . & . & . & $\mathrm{F}$ & $\mathrm{R}$ & . & & & . & . & V & . & & . & . & & . & & . & . & & & & \\
\hline $17-1.29$ & A & & P & & & & $\mathrm{F}$ & $\mathrm{R}$ & & & & & . & v & & & & & & & & & & . & & . & . \\
\hline $\mid 8-1,21$ & A & & P & & & & . & $\mathrm{F}$ & & . & & & . & . & & & & v & & $\mathrm{R}$ & & & & $\mathrm{R}$ & & & . \\
\hline $19-1,36$ & A & & $P$ & & & & v & F & & & & & & $\mathrm{F}$ & & & $\mathrm{F}$ & & & & & . & . & $\mathrm{R}$ & & . & \\
\hline $20-1,47$ & A & & $P$ & & & & V & V & & & & & & $\mathrm{R}$ & & & $\mathrm{R}$ & & & V & & v & & $\mathrm{R}$ & & . & \\
\hline $21-1.36$ & A & & $\mathrm{P}$ & & & & $\mathrm{F}$ & $\mathrm{R}$ & & & & & & $\mathrm{R}$ & & . & $\mathrm{R}$ & $\mathrm{R}$ & & $\mathrm{R}$ & & v & v & $\mathrm{R}$ & . & . & . \\
\hline $22-1,36$ & w & & $P$ & & & & $\mathrm{R}$ & $\mathrm{F}$ & & & V & C & & $\mathrm{R}$ & & & & $\mathrm{F}$ & . & V & . & & . & $\mathrm{R}$ & & . & \\
\hline $23-1,36$ & W & & $\mathrm{P}$ & & & & $\mathrm{F}$ & & & & & $\mathrm{F}$ & & $\mathrm{R}$ & & & & $\mathrm{R}$ & . & v & . & & . & $\mathrm{R}$ & & . & . \\
\hline $24-1,36$ & A & & $P$ & & & & $\mathrm{~F}$ & $\mathrm{R}$ & & & . & & & F & & . & $\mathrm{R}$ & $\mathrm{R}$ & . & $\mathrm{R}$ & & & . & $\mathrm{R}$ & v & . & . \\
\hline $24-6,36$ & W & & P & . & & . & F & $\mathrm{R}$ & & & & F & & $\mathrm{F}$ & & . & $\mathrm{R}$ & $\mathrm{F}$ & & v & & . & . & V & & . & . \\
\hline $26-1,36$ & A & & $\mathrm{P}$ & . & & . & $\mathrm{F}$ & F & . & & V & $\mathrm{F}$ & & F & . & & $\mathrm{R}$ & $\mathrm{F}$ & . & . & & & . & v & . & . & . \\
\hline $27-1,36$ & A & . & $P$ & & & & $\mathrm{~F}$ & $\mathrm{~F}$ & & & & $\mathrm{~F}$ & . & F & & & F & $\mathrm{F}$ & & $\mathrm{R}$ & & & . & $\mathrm{R}$ & & . & . \\
\hline $28-1,36$ & W & & $P$ & & & & R & $\mathrm{F}$ & & & V & $\mathrm{F}$ & . & F & & & $\mathrm{R}$ & $\mathrm{F}$ & & $\mathrm{R}$ & & & & V & & + & . \\
\hline $29-1,36$ & A & & $P$ & & & & $\mathrm{~F}$ & & & & V & F & & F & v & & v & v & . & v & . & v & . & $\mathrm{R}$ & & . & \\
\hline $30-1,36$ & A & & P & & & & $\mathrm{F}$ & & & & & C & & C & & . & R & $\mathrm{R}$ & . & $\mathrm{R}$ & . & . & . & & & . & \\
\hline $31-1,36$ & W & & $P$ & . & & & $\mathrm{C}$ & C & & & V & $\mathrm{R}$ & & C & & & $\mathrm{F}$ & $\mathrm{R}$ & . & $\mathrm{R}$ & . & & v & $\mathrm{R}$ & & . & . \\
\hline $32-1,36$ & W & & $P$ & & & & $\mathrm{~F}$ & F & & & . & $\mathrm{R}$ & & & & & F & $R$ & & $\mathrm{R}$ & & & & $\mathrm{F}$ & & . & \\
\hline $33-1.36$ & W & & $\mathrm{P}$ & & & & $\mathrm{F}$ & v & & & . & $\mathrm{R}$ & & $\mathrm{F}$ & & . & $\mathrm{R}$ & $\mathrm{F}$ & . & R & & & . & F & & & . \\
\hline $34-1,36$ & W & & M & . & & & $\mathrm{F}$ & v & & & . & V & & $\mathrm{F}$ & & & F & F & . & $\mathrm{R}$ & & & & v & . & . & . \\
\hline $35-1,36$ & W & & M & . & . & . & F & v & & . & . & V & . & R & & & R & $\mathrm{F}$ & & . & & & & $\mathrm{R}$ & . & . & . \\
\hline $36-1,35$ & w & & M & . & & v & F & F & . & & . & $\mathrm{R}$ & & . & . & & R & R & . & $\mathrm{V}$ & & & & $\mathrm{R}$ & . & . & . \\
\hline $37-1,35$ & W & & M & & & & $\mathrm{F}$ & $\mathrm{R}$ & & & . & V & . & $\mathrm{R}$ & . & . & $\mathrm{R}$ & $\mathrm{R}$ & & V & . & . & & $\mathrm{R}$ & . & . & . \\
\hline $38-1,37$ & W & & G & & . & & F & V & . & . & & $\mathrm{F}$ & & $\mathrm{F}$ & . & & $\mathrm{F}$ & $\mathrm{F}$ & & v & & V & & $\mathrm{F}$ & . & . & . \\
\hline $39-1,35$ & w & & G & & & & F & $\mathrm{R}$ & & & & F & & $\mathrm{F}$ & & & $\mathrm{R}$ & v & . & . & & & . & v & & . & A \\
\hline $40-1,37$ & W & & G & . & & . & F & . & . & . & & F & & $\mathrm{F}$ & & & F & $\mathrm{R}$ & C & $\mathrm{R}$ & & $\mathrm{R}$ & . & . & . & . & A \\
\hline $41-1,36$ & w & . & M & . & & & $\mathrm{F}$ & v & & & . & . & & . & & & V & . & C & . & & . & & v & . & . & $\mathrm{R}$ \\
\hline $42-1,39$ & W & . & M & . & & & $\mathrm{F}$ & & . & & . & $\mathrm{R}$ & & F & & & V & $\mathrm{F}$ & F & . & & v & & v & . & & $\mathrm{F}$ \\
\hline $43-1,39$ & W & . & P & . & . & & $\mathrm{F}$ & . & . & . & . & $\mathrm{F}$ & . & $\mathrm{R}$ & & R & V & & $\mathrm{F}$ & & & & & . & . & . & . \\
\hline $44-1,37$ & A & . & P & . & & & $\mathrm{R}$ & V & & & . & C & & V & & $\mathrm{F}$ & $\mathrm{R}$ & $\mathrm{v}$ & $\mathrm{F}$ & v & & $\mathrm{R}$ & & $\mathrm{R}$ & & & \\
\hline $45-1,36$ & W & & $P$ & & V & . & $\mathrm{R}$ & & $\mathrm{R}$ & & $\therefore$ & $\mathrm{F}$ & . & C & . & $\mathrm{R}$ & & & $\mathrm{F}$ & . & & & & $\mathrm{v}$ & . & . & . \\
\hline $46-1,36$ & C & & P & & & & $\mathrm{R}$ & & V & & . & $\mathrm{R}$ & & $\mathrm{R}$ & & & & . & V & $\cdot$ & . & . & & . & . & . & . \\
\hline $47-1.36$ & C & & $P$ & & . & & v & & . & & . & $\mathrm{F}$ & & $\mathrm{R}$ & . & & . & & C & . & & & & v & . & . & . \\
\hline $48-1,12$ & C & & $\mathrm{p}$ & & . & & v & . & & . & . & . & . & $\mathrm{F}$ & . & & & & . & & . & & & . & . & . & . \\
\hline
\end{tabular}


Table 1 (continued).

\begin{tabular}{|c|c|c|c|c|c|c|c|c|c|c|c|c|c|c|c|c|c|c|c|c|c|c|c|c|c|c|c|}
\hline & 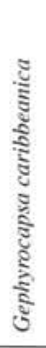 & 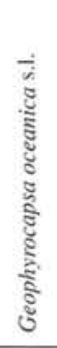 & 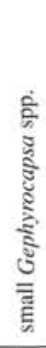 & 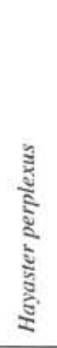 & 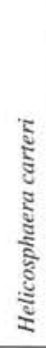 & 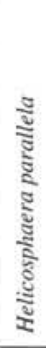 & 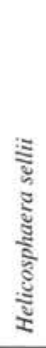 & 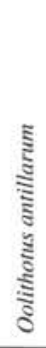 & 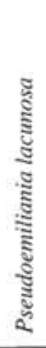 & 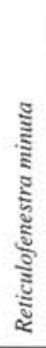 & 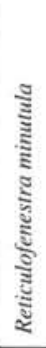 & 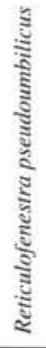 & 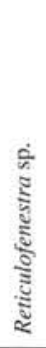 & 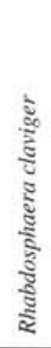 & है & 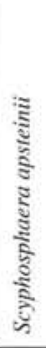 & 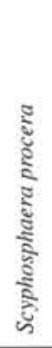 & 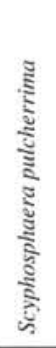 & 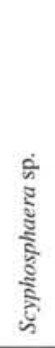 & 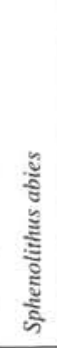 & 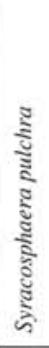 & 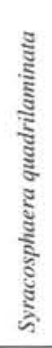 & 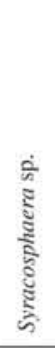 & 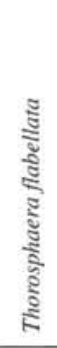 & 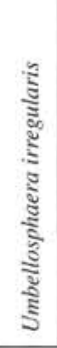 & 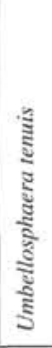 & 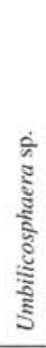 \\
\hline $9-4,37$ & & & C & . & $\mathrm{F}$ & . & R & . & C & A & A & . & & F & v & & & . & . & . & F & & & & . & & \\
\hline 5,37 & & & $\mathrm{R}$ & . & F & & . & . & F & A & A & . & & & v & & v & . & . & & F & & & & . & & \\
\hline $9-6,37$ & . & $\therefore$ & $\mathrm{R}$ & . & $\mathrm{F}$ & & & & F & C & A & . & & R & $\mathrm{R}$ & & . & . & . & & V & . & . & . & . & & \\
\hline $9-7.37$ & . & & R & . & $\mathrm{R}$ & & . & . & $\mathrm{F}$ & A & A & . & & & . & . & & $\cdot$ & & . & v & & . & & . & & \\
\hline $10-1,36$ & . & & A & . & $\mathrm{R}$ & & v & , & A & W & W & . & & V & $\mathrm{R}$ & & . & . & & & $\mathrm{R}$ & & . & . & . & & \\
\hline 10-2, & . & , & C & . & $\mathrm{F}$ & . & $\therefore$ & & $\mathrm{F}$ & A & A & . & & V & & V & v & & & . & $\mathrm{R}$ & . & & . & . & & . \\
\hline $10-3,36$ & . & & C & . & $\mathrm{F}$ & & V & & $\mathrm{F}$ & A & A & . & & . & V & & & . & . & . & & & & & & . & . \\
\hline $10-4,36$ & . & . & C & . & C & & & & C & A & A & . & & . & . & & & . & & & V & & & . & . & & v \\
\hline $10-5,36$ & $\cdot$ & & & . & F & & . & . & C & A & A & . & & & v & & & . & . & & v & . & & . & . & & \\
\hline $10-6,36$ & . & . & C & v & F & & & & C & A & A & . & & $\mathrm{R}$ & . & & & & & & V & . & & . & . & & . \\
\hline $10-7.36$ & . & . & C & . & $\mathrm{F}$ & & & & C & A & A & . & & & $\mathrm{R}$ & & & & & & . & . & . & . & . & & \\
\hline $11-1,36$ & . & . & C & . & $\mathrm{F}$ & & . & & C & & A & . & & . & V & & & . & & & R & . & . & & . & & \\
\hline $11-2,36$ & . & . & C & & $\mathrm{F}$ & & . & . & F & C & A & . & & & V & & & & & & . & . & , & & . & & \\
\hline $11-3,36$ & . & & A & . & $\mathrm{F}$ & & . & & A & & A & . & . & v & . & . & & . & & & . & . & $\cdot$ & & . & & \\
\hline $11-4,36$ & . & . & . & . & $\mathrm{F}$ & . & & & $\mathrm{F}$ & . & A & & . & . & v & & & . & & & . & , & . & . & . & . & . \\
\hline 5,36 & . & . & A & . & $\mathrm{F}$ & . & . & . & $\mathrm{F}$ & A & A & & . & $\mathrm{R}$ & . & & . & . & & & & . & . & . & . & 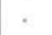 & . \\
\hline $11-6,36$ & . & . & C & . & F & . & . & . & $\mathrm{F}$ & A & A & . & & $\mathrm{R}$ & . & & & . & . & V & $\mathrm{R}$ & . & . & . & . & . & . \\
\hline $11-7.36$ & . & . & W & & F & . & & . & C & . & A & & & $\mathrm{R}$ & V & & . & . & . & V & $\mathrm{R}$ & . & . & . & . & & . \\
\hline $12-1,36$ & $\cdot$ & $\because$ & W & . & F & & & . & C & . & A & & & $\mathrm{R}$ & V & & v & . & . & v & R & . & $\cdot$ & $\cdot$ & . & . & . \\
\hline $12-2,36$ & . & & A & v & F & & . & . & C & A & A & & & V & v & 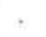 & . & . & . & V & $\mathrm{F}$ & . & . & . & . & & . \\
\hline$-1,36$ & . & & C & & $\mathrm{F}$ & & . & . & C & . & A & . & & & . & & . & . & . & $\mathrm{R}$ & & . & . & & & . & . \\
\hline $14-1,36$ & & & A & & $\mathrm{F}$ & & V & . & C & . & A & . & & $\mathrm{R}$ & v & & & . & . & F & $\mathrm{R}$ & , & . & & . & & \\
\hline $15-1,36$ & . & & A & & $\mathrm{F}$ & & F & V & C & A & A & . & & & & & . & & . & F & R & . & . & . & . & & \\
\hline $16-1,36$ & . & & w & . & F & & $\mathrm{R}$ & . & A & A & A & & & R & v & . & & . & . & F & $\mathrm{R}$ & . & . & & . & . & . \\
\hline $17-1$, & & & A & . & $\mathrm{F}$ & & $\mathrm{R}$ & . & A & A & A & & & $\mathrm{R}$ & V & & & & & F & & & & & & & . \\
\hline & & & A & . & C & & . & . & F & . & $A$ & $\mathrm{R}$ & & & . & & & . & & C & & & & . & . & & . \\
\hline$-1,36$ & . & . & w & v & $\mathrm{F}$ & & $\mathrm{R}$ & . & $\mathrm{F}$ & & A & $\mathrm{F}$ & & v & & & & & . & C & 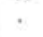 & . & . & . & . & . & . \\
\hline $20-1,47$ & . & . & A & & C & & $\mathrm{R}$ & . & F & A & A & F & & $\mathrm{R}$ & . & & & . & . & C & . & . & . & . & . & . & . \\
\hline $21-1,36$ & . & . & A & . & $\mathrm{F}$ & & v & . & . & . & $\mathrm{R}$ & R & . & . & v & & & . & . & C & $\mathrm{R}$ & + & $\cdot$ & . & . & . & \\
\hline $22-1,3 t$ & . & & W & & C & & $\mathrm{R}$ & . & F & A & A & F & & & V & & & & & A & & & & . & . & & . \\
\hline 2. & . & . & C & & $\mathrm{F}$ & . & V & . & $\mathrm{R}$ & A & A & $\mathrm{F}$ & & & . & & & 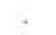 & . & & & . & . & . & . & & \\
\hline 24 & . & & A & & $\mathrm{F}$ & & v & . & R & A & A & R & & $\mathrm{R}$ & V & & & & & C & . & . & . & & . & . & . \\
\hline $24-6,3$ & . & & A & & C & & $\mathrm{R}$ & & F & A & A & F & & & . & & & . & & C & & . & . & & . & . & \\
\hline $26-1,36$ & . & . & C & . & $\mathrm{F}$ & . & . & . & C & A & A & $\mathrm{F}$ & & $\mathrm{R}$ & v & . & & . & . & C & v & . & . & . & . & & . \\
\hline 27 & . & . & A & . & C & . & & & C & A & & $\mathrm{F}$ & & & . & & & & . & C & & . & . & . & . & & . \\
\hline 2 & & . & C & . & $\mathrm{F}$ & & & . & C & W & A & $\mathrm{F}$ & & $\mathrm{F}$ & & & & & . & c & & . & . & . & . & 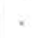 & . \\
\hline & & . & C & . & $\mathrm{F}$ & & & . & C & A & A & $\mathrm{F}$ & & & & & & & . & C & & . & . & . & . & & \\
\hline $30-1,36$ & & & C & & $\mathrm{F}$ & & $\mathrm{v}$ & . & $\mathrm{F}$ & A & A & F & & $R$ & . & & & $\theta^{\circ}$ & . & C & & & & & & . & \\
\hline $31-1,36$ & . & . & C & . & C & & V & . & C & W & A & F & & V & . & & . & & . & C & $\mathrm{v}$ & . & . & & . & & \\
\hline & & & $\mathrm{R}$ & & C & & . & . & $\mathrm{F}$ & W & & F & & & v & & 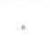 & . & . & & & & . & & . & & . \\
\hline & & . & & & & & & . & $\mathrm{F}$ & $v$ & C & F & . & & & & & . & . & & & . & . & & . & 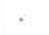 & . \\
\hline 3 & 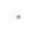 & & & . & C & & & . & F & n & . & C & & R & . & & 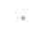 & & . & C & & . & . & & . & . & \\
\hline & & & . & . & F & & & & & w & W & A & & $\mathrm{R}$ & & & & & . & C & . & . & & . & . & . & . \\
\hline $36-1,35$ & & $\therefore$ & . & . & C & . & & & . & W & . & A & & $\mathrm{R}$ & v & & & & . & C & v & . & . & . & . & & \\
\hline & & . & . & . & C & & & . & . & u & . & A & & 1 & v & & & & . & A & & & . & . & . & & \\
\hline & & . & . & . & $c$ & $\mathrm{R}$ & & . & & v & . & A & & 1 & 1 & & & v & . & A & F & . & & & . & & \\
\hline 3 & & . & . & . & $\mathrm{F}$ & & & . & . & u & A & A & & $\mathrm{F}$ & v & & . & . & . & A & . & + & . & & . & & \\
\hline & & & . & . & $\mathrm{F}$ & & & . & . & W & A & A & & F & V & & & & . & A & & & & & . & . & \\
\hline $41-1,36$ & & & . & . & C & & . & . & . & W & A & C & & $\mathrm{R}$ & & & . & & . & A & & . & . & . & . & & . \\
\hline & & & . & . & & & V & , & . & & A & $c$ & & $v$ & & & . & & . & & & & & & & & \\
\hline & & & . & . & $c$ & & . & . & . & u & . & c & & & V & & . & . & . & & & & & & & & \\
\hline & & & & & c & $\mathrm{P}$ & . & & . & A & 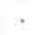 & A & & F & . & & . & . & . & A & & & & . & . & & . \\
\hline & & & & . & C & $\mathrm{R}$ & . & . & & W & . & C & & $\mathrm{R}$ & V & & . & . & . & A & & & & & . & . & \\
\hline $46-1,36$ & . & . & v & . & V & . & . & & & $\mathrm{F}$ & v & . & & . & & . & & . & , & C & . & . & & & . & & . \\
\hline & & & . & . & V & & & . & . & & &. & & & & & & & & & & & & & . & & \\
\hline $48-1,12$ & . & . & . & . & & . & . & & & C & $\mathrm{R}$ & . & & & . & & & . & & C & & & & & . & & \\
\hline
\end{tabular}




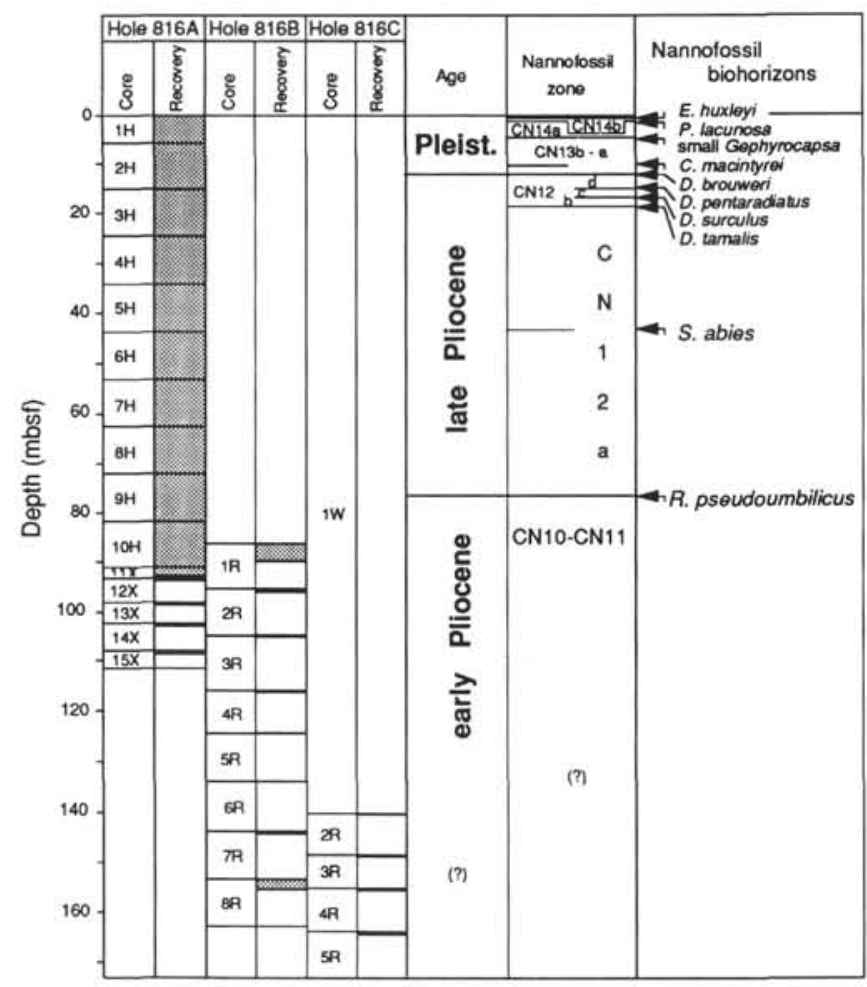

Figure 8. Summary of nannofossil biostratigraphy for Site 816.

of Site 817 has been revised significantly from that given in the Leg 133 Initial Reports volume, which is not surprising, given the large amount of redeposition that is associated with the turbidites and slumps encountered in the sediments. The biostratigraphy of the site is summarized in Figure 9, and a complete checklist for the site is given in Table 2 (back pocket).

The youngest biohorizon recognizable is the lower limit of the Emiliania huxleyi Acme at $7.58 \mathrm{mbsf}(817 \mathrm{~A}-2 \mathrm{H}-2,38 \mathrm{~cm})$, which level has an age of only about $0.075 \mathrm{Ma}$ and, thus, indicates a sedimentation rate of close to $100 \mathrm{~m} / \mathrm{m}$.y. for the Pleistocene and Holocene interval. The lowest occurrence of Emiliania huxleyi is at $25.07 \mathrm{mbsf}(817 \mathrm{~A}-4 \mathrm{H}-1,37 \mathrm{~cm})$, which datum has an age of $0.275 \mathrm{Ma}$ and indicates that the high sedimentation rate commenced at least that far back. The next lower biohorizon, the highest occurrence of Pseudoemiliania lacunosa is only a short distance lower at $28.07 \mathrm{mbsf}(817 \mathrm{~A}-4 \mathrm{H}-3,37 \mathrm{~cm})$ and, because this datum has an age of $0.465 \mathrm{Ma}$, the sedimentation rate between this and the next higher biohorizon is much lower-only 10 to $15 \mathrm{~m} / \mathrm{m}$.y. Possibly a hiatus is present between the last two biohorizons. The small Gephyrocapsa dominance interval extends from $45.56(817 \mathrm{~A}-6 \mathrm{H}-2,36 \mathrm{~cm})$ to $51.46 \mathrm{mbsf}(817 \mathrm{~A}-6 \mathrm{H}-6,36 \mathrm{~cm})$, and highest occurrence of Calcidiscus macintyrei is at $63.06 \mathrm{mbsf}(817 \mathrm{~A}-8 \mathrm{H}-1,36 \mathrm{~cm})$.

Several biohorizons cluster at $72.56 \mathrm{mbsf}(817 \mathrm{~A}-9 \mathrm{H}-1,36 \mathrm{~cm})$, including the lowest occurrence of large specimens of Gephyrocapsa (= Gephyrocapsa spp.), and the highest occurrences of Discoaster brouweri, Discoaster pentaradiatus, and Discoaster surculus. The first and the last biohorizons have ages of 1.59 and $2.43 \mathrm{Ma}$, respectively, which indicates that there must be a hiatus at this level that spans at least $0.84 \mathrm{~m}$.y. Clearly, turbidity currents removed, as well as deposited, sediments at this location. The highest occurrence of Discoaster tamalis is slightly lower at $75.56 \mathrm{mbsf}(817 \mathrm{~A}-9 \mathrm{H}-3,36 \mathrm{~cm})$.

The highest occurrence of Sphenolithus abies is at $155.70 \mathrm{mbsf}$ ( $817 \mathrm{~A}-17 \mathrm{H}-5,150 \mathrm{~cm})$, which is also the highest occurrence of Reticulofenestra pseudoumbilicus. Although these two biohorizons are separated by only a short time interval, they do not coincide, and the close correspondence may indicate that a minor hiatus is present at this level.
The next lower biohorizon is the lowest occurrence of Discoaster tamalis at $158.06 \mathrm{~cm}(817 \mathrm{~A}-18 \mathrm{H}-2,36 \mathrm{~cm})$, followed shortly by the lowest occurrence of Pseudoemiliania lacunosa and the highest occurrence of Amaurolithus tricorniculatus at $161.06 \mathrm{mbsf}$ (817A-18H$3,36 \mathrm{~cm}$ ). Birefringent ceratoliths (Ceratolithus armatus and Ceratolithus rugosus) occur downward to $176.70 \mathrm{mbsf}(817 \mathrm{~A}-19 \mathrm{H}-$ CC), and the consistent occurrence of Amaurolithus tricorniculatus downward to this same level suggests that there is probably a hiatus in the lower Pliocene interval. The highest occurrence of Discoaster quinqueramus (i.e., the Miocene/Pliocene boundary) is just a short distance below at $180.06 \mathrm{mbsf}(817 \mathrm{~A}-20 \mathrm{H}-3,37 \mathrm{~cm})$.

Upper Miocene biohorizons include the highest occurrence of Amaurolithus amplificus at $186.07 \mathrm{mbsf}(817 \mathrm{~A}-20 \mathrm{H}-7,37 \mathrm{~cm})$, the highest occurrence of Discoaster berggrenii at 189.57 mbsf (817A$21 \mathrm{X}-3,37 \mathrm{~cm}$ ), the lowest occurrence of Amaurolithus amplificus at $195.57 \mathrm{mbsf}(817 \mathrm{~A}-21 \mathrm{H}-7,37 \mathrm{~cm})$, and the lowest occurrence of Amaurolithus primus at $200.56 \mathrm{mbsf}(817 \mathrm{~A}-22 \mathrm{H}-4,36 \mathrm{~cm})$. The lowest occurrence of Discoaster quinqueramus is at $205.20 \mathrm{mbsf}$ ( $817 \mathrm{~A}-22 \mathrm{H}-\mathrm{CC})$, which suggests that the section between the last two biohorizons has been shortened by a significant hiatus. The section below the last datum is chalky and yielded a more generalized assemblage that is not suitable for precise zonation of the interval, although four additional biohorizons can be identified.

The highest occurrence of Coccolithus miopelagicus is at 227.76 mbsf (817A-25X-3, $36 \mathrm{~cm})$; this is probably the closest datum for marking the middle Miocene to late Miocene transition. The highest occurrence of Cyclicargolithus floridanus is at $234.0 \mathrm{mbsf}$ (817A$25 \mathrm{X}-\mathrm{CC}$ ); the highest occurrence of Calcidiscus premacintyrei is at $259.66 \mathrm{mbsf}(817 \mathrm{~A}-28 \mathrm{X}-5,36 \mathrm{~cm})$; and the lowest biohorizon, the highest occurrence of Discoaster exilis, is at $272.70 \mathrm{mbsf}$ (817A29X-CC). The last two biohorizons, and particularly the highest occurrence of Discoaster exilis, may not be entirely reliable because they are based on relatively scarce occurrences of the markers in a generally unfavorable lithology.

\section{Site $\mathbf{8 1 8}$}

Site 818 also is located on the north side of Townsville Trough, east of Site 817 (see Fig. 1). The biostratigraphic summary for this site is given in Figure 10. Calcareous nannofossils are abundant, except at the very bottom of the cored interval, but preservation is highly variable, from near pristine at the top of the section to excessively calcified and overgrown in sandy and chalky intervals. The succession of biohorizons at this site is similar to the succession at Site 817 , especially for the Pleistocene and the latest Pliocene, although the section is more expanded.

The youngest biohorizon recognized is the lowest occurrence of Emiliania huxleyi at $8.78 \mathrm{mbsf}(818 \mathrm{~B}-2 \mathrm{H}-1,38 \mathrm{~cm})$, followed by the highest occurrence of Pseudoemiliania lacunosa at $14.7 \mathrm{mbsf}$ (818B$2 \mathrm{H}-5,38 \mathrm{~cm})$. The spacing of these two biohorizons suggests a more or less constant sedimentation rate of about $31 \mathrm{~m} / \mathrm{m}$.y. for the latest Pleistocene (i.e., the last 0.5 m.y.). The next lower biohorizon is the top of the interval of small Gephyrocapsa dominance at $52.76 \mathrm{mbsf}$ $(818 \mathrm{~B}-6 \mathrm{H}-5,36 \mathrm{~cm})$, which is much lower than would be projected from the previous two biohorizons. Next below can be identified the highest occurrence of Calcidiscus macintyrei at $79.76 \mathrm{mbsf}$ (818B$9 \mathrm{H}-4,36 \mathrm{~cm}$ ), followed by the simultaneous highest occurrences of Discoaster brouweri and Discoaster pentaradiatus at $84.77 \mathrm{mbsf}$ (818B-10H-1, $37 \mathrm{~cm})$. The co-occurrence of these two markers suggests a significant hiatus in the vicinity of the Pliocene/Pleistocene boundary, as was true at Site 817 also. The upper Pliocene section at Site 818 is more expanded and more complete than that at Site 817. The highest occurrence of Discoaster surculus is at $105.26 \mathrm{mbsf}$ (818B-12H-1, $36 \mathrm{~cm})$, followed by the highest occurrence of Discoaster tamalis at 176.27 mbsf $(818 \mathrm{~B}-19 \mathrm{H}-5,37 \mathrm{~cm})$, the highest occurrence of Sphenolithus abies at 246.26 mbsf (818B-27H-1, $36 \mathrm{~cm})$, and the highest occurrence of Reticulofenestra pseudoum- 


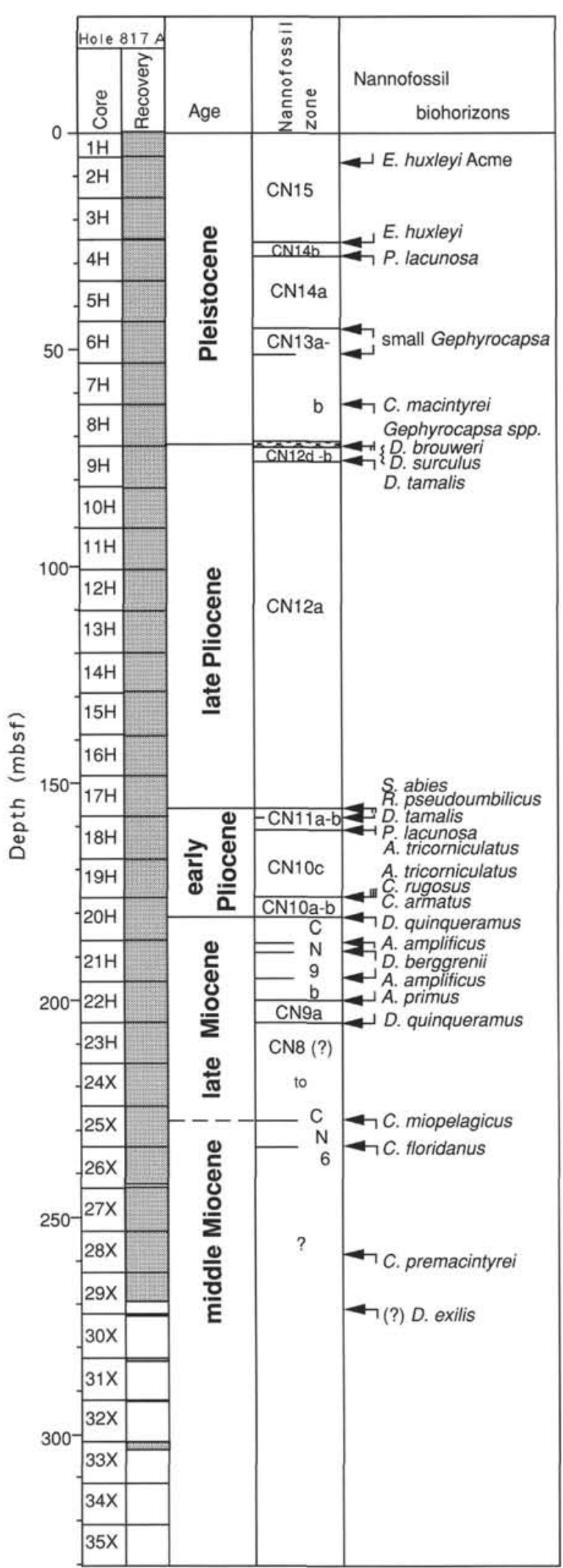

Figure 9. Summary of nannofossil biostratigraphy for Site 817. bilicus at $258.76 \mathrm{mbsf}(818 \mathrm{~B}-28 \mathrm{H}-3,36 \mathrm{~cm})$. Thus, the upper Pliocene section is about twice as thick at this site as that at the nearby Site 817.

No further biohorizons were identified at this site and the remaining $40 \mathrm{~m}$ of the section (through Core $32 \mathrm{H}$ ) has been assigned a latest early Pliocene age.

\section{BIOSTRATIGRAPHIC PROBLEMS AND PROSPECTS}

The location of Leg 133 drilling - moderate depth tropical oceans, expanded sections of hemipelagic sediments-makes this material a particularly attractive subject for further study. The abundance and diversity of calcareous nannofossils permitted precision in the dating of sediments, limited only by the distance separating adjacent samples and by the time available for processing the large number of samples. Nevertheless, other important constraints exist. As elsewhere, preservation of calcareous nannofossils is best in clay-rich sediments and tends to deteriorate rapidly downcore in sediments in which the pelagic component is diluted mainly by platform-derived, shallow-water carbonates. Where shallow-water carbonate deposition dominated, nannofossil recovery becomes insufficient for dating. The upper Miocene marker Discoaster quinqueramus proved useful in marginally preserved assemblages because, even in an overgrown or corroded state, this species can be identified with some confidence. The middle Miocene Sphenolithus heteromorphus, Cyclicargolithus floridanus, and, surprisingly, Calcidiscus premacintyrei were encountered most often in otherwise unpromising shallow-water carbonates.

The lack of a usable magnetic stratigraphy in the Leg 133 sediments is disappointing in that these sediments will not be useful for refining nannofossil biochronology. Combining the biostratigraphy with isotope stratigraphy may ultimately prove more tractable.

Figure 11 (back pocket) is a diagrammatic representation of the sites from the southern transect of Townsville Trough. An age scale is given on the two extremes of the illustration; the seven sites from north and south of Townsville Trough are represented as depth sections. Dashed lines connect the sites and the time scales at the Pliocene/Pleistocene boundary, at the late Pliocene/early Pliocene boundary (= Reticulofenestra pseudoumbilicus highest occurrence), and at the Miocene/Pliocene boundary. The dramatic differences in sedimentation rate in the hemipelagic interval at adjacent sites can be seen clearly and traced in this representation.

\section{HIATUSES AND EXPANDED INTERVALS}

Table 3 is a listing of the ages and depths of the various biohorizons identified at Sites 812 through 818 . Age/depth plots of all of these data are given in a single graph in Figure 12. Combining the several representations of the data, the following generalizations can be offered. At Sites 812,813 , and 814 , the three shallow sites on the southern flank of Queensland Plateau, pelagic sedimentation became significant just below the highest occurrence of Discoaster quinqueramus (i.e., immediately preceding the end of the Miocene). The precise timing can be constrained to between 5.26 and $5.6 \mathrm{Ma}$, the highest occurrences, respectively, of Discoaster quinqueramus and Amaurolithus amplificus. At two of these sites, 813 and 814, the transition is marked by a distinct hiatus. This hiatus encompasses much of the lower Pliocene at Site 814, and all but the uppermost part of the lower Pliocene at Site 813. Conceivably, part of the uppermost Miocene may be included as well. Nondeposition, probably caused by currents impinging on the southern edge of Queensland Plateau, is the preferred interpretation, as no significant lithologic change is associated with this hiatus.

The onset of current activity may be associated with local events or may be connected with the major reorganization of ocean circulation and/or sea-level changes known to have occurred toward the end of the Miocene. As these sites descended progressively below the effective depth of surface currents, pelagic sediments and periplat- 


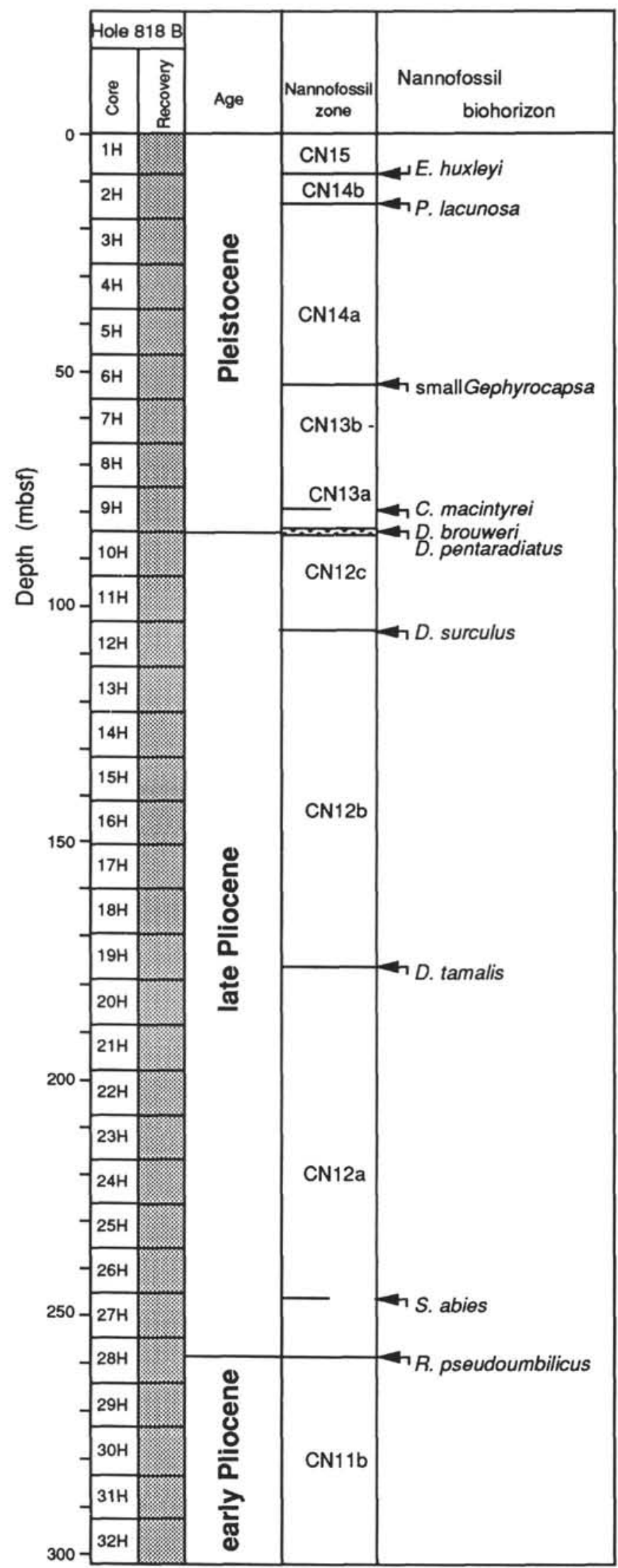

Figure 10. Summary of nannofossil biostratigraphy for Site 818 . form oozes commenced to accumulate, although the timing and rate may have been influenced also by local topography. Site 812 is significantly shallower and behind a positive topographic feature, and this may have kept it from experiencing similar erosion, although biostratigraphic resolution is not good enough to say this with certainty. At all three sites the latest Pliocene through Pleistocene record is without detectable hiatuses, and a stable sedimentary regime, much like today's, seems to have prevailed for about the past 2.5-3.5 m.y.

The sediments at Sites 817 and 818, the two deeper sites on the southern edge of Queensland Plateau, have a more pelagic aspect; consequently, a more detailed biostratigraphy could be extracted. At Site 817 a minor episode of nondeposition or deep current erosion is indicated in the lower Pliocene by the correspondence of three biohorizons, the lowest occurrences of Amaurolithus tricorniculatus, Ceratolithus rugosus, and Ceratolithus armatus, and in the mid-Pliocene by the correspondence of the highest occurrence of Reticulofenestra pseudoumbilicus and Sphenolithus abies. The lowermost Pliocene was not penetrated at Site 818 , and the mid-Pliocene shows no evidence of a hiatus there. Both sites, however, have a significant hiatus straddling the Pliocene/Pleistocene boundary. At both sites the hiatus extends only into the lowermost Pleistocene, but nondeposition is manifested somewhat lower at Site 817 than at Site 818 (2.6 Ma vs. $\sim 2.8 \mathrm{Ma}$ ) This may be explained by the fact that at Site 818 late Pliocene-age deposition occurred in a depression, a channel formed during an earlier erosional episode. No further hiatuses are apparent in the Pleistocene.

In contrast to the sites on the north side of Townsville Trough (southern flank of Queensland Plateau), Sites 815 and 816, cored on the south side of the trough (northern flank of Marion Plateau), have no discernable hiatuses within the interval represented by hemipelagic sediments (i.e., the upper Miocene to Holocene for Site 815 and the mid-Pliocene to Holocene for Site 816). At both sites, however, the mid-Pliocene section is greatly expanded relative to older sediments at Site 815 , and relative to younger sediments at both sites. When this expanded section is examined on seismic sections (Davies, McKenzie, Palmer-Julson, et al., 1991), it is clear that much of this sediment was redeposited, probably by contour currents. Remarkably, redeposition appears to have involved only contemporaneous sediment, as virtually no redeposited nannofossils are evident within the expanded interval.

The data base (number of sites cored) is almost certainly too small for this complex area, and the site selection no doubt has a built-in bias, so that generalizations about hiatuses and expanded sections on the flanks of Townsville Trough probably are not justified. Clearly, bottom currents have had a significant role in rearranging of hemipelagic sediments in Townsville Trough. Within limited areas, distinct episodes of erosion and of deposition can be recognized. Moreover, currents have been sufficiently intense to prevent deposition of hemipelagic sediments of the entire spectrum of particle sizes, but not strong enough to scour and erode older hemipelagic deposits.

\section{ACKNOWLEDGMENTS}

This manuscript has benefited measurably from thoughtful and thorough reviews by D. Watkins, E. de Kaenel, A. Palmer-Julson, and S. Stewart. Their efforts are happily acknowledged.

\section{REFERENCES}

Baldauf, J.G., Thomas, E., Clement, B., Takayama, T., Weaver, P.P.E., Backman, J., Jenkins, G., Mudie, P.J., and Westberg-Smith, M.J., 1987. Magnetostratigraphic and biostratigraphic synthesis, Deep Sea Drilling Project Leg 94. In Ruddiman, W.F., Kidd, R.B., et al., Init. Repts. DSDP, 94 (Pt. 2): Washington (U.S. Govt. Printing Office), 1159-1205.

\footnotetext{
Abbreviations for names of organizations and publications in ODP reference lists follow the style given in Chemical Abstracts Service Source Index (published by American Chemical Society).
} 
Berggren, W.A., Kent, D.V., and Van Couvering, J.A., 1985. The Neogene: Part 2. Neogene geochronology and chronostratigraphy. In Snelling, N.J. (Ed.), The Chronology of the Geological Record. Geol. Soc. London Mem., 10:211-260.

Davies, P.J., McKenzie, J.A., Palmer-Julson, A., et al., 1991. Proc. ODP, Init. Repts., 133: College Station, TX (Ocean Drilling Program).

Gartner, S., 1992. Miocene nannofossil chronology in the North Atlantic, DSDP Site 608. Mar. Micropaleontol., 18:307-331.

Martini, E., 1971. Standard Tertiary and Quaternary calcareous nannoplankton zonation. In Farinacci, A. (Ed.), Proc. 2nd Int. Conf. Planktonic Microfossils Roma: Rome (Ed. Tecnosci.), 2:739-785.

Okada, H., and Bukry, D., 1980. Supplementary modification and introduction of code numbers to the low-latitude coccolith biostratigraphic zonation (Bukry, 1973; 1975). Mar. Micropaleontol., 5:321-325.

Date of initial receipt: 16 March 1992

Date of acceptance: 10 November 1992

Ms 133SR-213

\section{APPENDIX}

List of species mentioned in the text, arranged in alphabetical order by generic epithets.

Amaurolithus amplificus (Bukry and Percival, 1971) Gartner and Bukry, 1975

Amaurolithus bizzarus (Bukry, 1973) Gartner and Bukry, 1975

Amaurolithus delicatus Gartner and Bukry, 1975

Amaurolithus primus (Bukry and Percival, 1971) Gartner and Bukry, 1975

Amaurolithus tricorniculatus (Gartner, 1967) Gartner and Bukry, 1975

Braarudosphaera bigelowi (Gran and Braarud, 1935) Deflandre, 1947

Calcidiscus leptoporus (Murray and Blackman, 1898) Loeblich and Tappan, 1978

Calcidiscus macintyrei (Bukry and Baramlette, 1969) Loeblich and Tappan, 1978

Calcidiscus pataecus (Gartner, 1967)

Coccolithus pataecus Gartner, 1967

Calcidiscus premacintyrei Theodorides, 1984

Calcidiscus tropicus Kamptner, 1955 (see Gartner, 1992)

Ceratolithus acutus Gartner and Bukry, 1974

Ceratolithus cristatus Kamptner, 1950

Ceratolithus rugosus Bukry and Baramlette, 1969

Ceratolithus telesmus Norris, 1965

Coccolithus minimus Kamptner, 1963

Coccolithus miopelagicus Bukry, 1971

Coccolithus pelagicus (Wallich, 1877) Schiller, 1930

Coronocyclus nitescens (Kamptner, 1963) Bramlette and Wilcoxon, 1967

Cricosphaera quadrilaminata Okada and McIntyre, 1977

Cryptococcolithus takayamae Gartner, 1992

Cyclicargolithus floridanus (Roth and Hay, 1967) Bukry, 1971

Cyclococcolithus cricotus Gartner, 1967

Cyclolithella annula (Cohen, 1964) Boudreaux and Hay, 1969

Dictyococcites antarcticus Haq, 1976

Dictyococcites productus (Kamptner, 1963) Backman, 1980

Discoaster adamanteus Bramlette and Wilcoxon, 1967

Discoaster asymmetricus Gartner, 1969

Discoaster berggrenii Bukry, 1971

Discoaster blackstockae Bukry, 1973

Discoaster brouweri Tan, 1927

Discoaster challangeri Bramlette and Riedel, 1954

Discoaster deflandrei Bramlette and Riedel, 1954

Discoaster druggii Bramlette and Wilcoxon, 1967

Discoaster exilis Martini and Bramlette, 1963

Discoaster formosus Martini and Worsley, 1971

Discoaster intercalaris Bukry, 1971

Discoaster loeblichii Bukry, 1971

Discoaster moorei Bukry, 1971

Discoaster neohamatus Bukry and Bramlette, 1969
Discoaster neorectus Bukry, 1971

Discoaster pansus (Bukry and Percival, 1971) Bukry, 1973

Discoaster pentaradiatus Tan, 1927

Discoaster quadramus Bukry, 1973

Discoaster quinqueramus Gartner, 1969

Discoaster surculus Martini and Bramlette, 1963

Discoaster tamalis Kamptner, 1967

Discoaster triradiatus Tan, 1927

Discoaster variabilis Martini and Bramlette, 1963

Discolithina discopora $($ Schiller, 1925) $=$ Pontosphaera discopora Schiller, 1925

Discolithina indooceanica $($ Cepek, 1973) $=$ Pontosphaera indooceanica Cepek, 1973

Discolithina japonica Takayama, 1967

Discolithina multipora Kamptner, 1948

Discolithina rimosa Bramlette and Sullivan, 1961

Discolithina scutellum $($ Kamptner, 1952) = Pontosphaera scutellum Kamptner, 1952

Discolithina syracusana $($ Lohmann, 1902) = Pontosphaera syracusana Lohmann, 1902

Discosphaera tubifer (Murray and Blackman, 1898) Ostenfeld, 1900

Emiliania huxleyi (Lohman, 1902) Hay and Mohler, 1967

Florisphaera profunda Okada and Honjo, 1973

Gephyrocapsa caribbeanica Boudreaux and Hay, 1969

Gephyrocapsa margerelli Breheret, 1978

Gephyrocapsa muellerae Breheret, 1978

Gephyrocapsa oceanica Kamptner, 1943

Hayaster perplexus (Bramlette and Ridel, 1953) Bukry, 1973

Helicosphaera carteri (Wallich, 1877) Kamptner, 1954

Helicosphaera euphrates Haq, 1966

Helicosphaera hyalina Gaarder, 1970

Helicosphaera inversa Gartner, 1980

Helicosphaera obliqua (Bramlette and Wilcoxon, 1967)

Helicosphaera orientalis Black, 1971

Helicosphaera parallela (Bramlette and Wilcoxon, 1967)

Helicosphaera sellii Bukry and Bramlette, 1969

Helicosphaera wallichi (Lohmann, 1902) Boudreaux and Hay, 1969

Holodiscolithus macroporus (Deflandre, 1954) Roth, 1970

Oolithotus antillarum (Cohen, 1964) Reinhardt, 1968

Oolithotus fragilis (Lohmann, 1912) Martini and Mueller, 1972

Pseudoemiliania lacunosa (Kamptner, 1963) Gartner, 1969

Reticulofenestra minuta Roth, 1970

Reticulofenestra minutula (Gartner, 1967) Haq and Berggren, 1978

Reticulofenestra pseudoumbilicus (Gartner, 1967) Gartner, 1969

Rhabdosphaera claviger Murray and Blackman, 1898

Rhabdosphaera longistylis Schiller, 1925

Rhabdosphaera procera Martini,1969

Rhabdosphaera stylifer Lohmann, 1902

Scapholithus fossilis Deflandre, 1954

Scyphosphaera apsteinii Lohmann, 1902

Scyphosphaera pulcherrima Deflandre, 1942

Sphenolithus abies Deflandre, 1954

Sphenolithus moriformis (Bronnimann and Stradner, 1960) Bramlette and Wilcoxon, 1967

Syracosphaera binodata Kamptner, 1937

Syracosphaera histrica Kamptner, 1941

Syracosphaera lamina Lecal-Schlauder, 1951

Syracosphaera nodosa Kamptner, 1941

Syracosphaera pulchra Lohmann, 1902

Thoracosphaera fossata Jafar, 1975

Thoracosphaera hemii (Lohmann, 1919) Kamptner, 1941

Thoracosphaera saxea Stradner, 1961

Thorosphaera flabellata Halldal and Markali, 1955

Triquetrorhabdulus rugosus Bramalette and Wilcoxon, 1967

Umbilicosphaera dilatata Kamptner, 1963

Umbilicosphaera hulburtiana Gaarder, 1955

Umbilicosphaera lexus $($ Kamptner, 1963) = Tiarolithus laxus Kamptner, 1963

Umbilicosphaera sibogae (Weber-van Bosse, 1901) Gaarder, 1970

Umbellosphaera irregularis Paasche, 1955

Umbellosphaera tenuis (Kamptner, 1937) Paasche, 1955 
Table 3. Calcareous nannofossil biohorizons, Sites 812-818.

\begin{tabular}{|c|c|c|c|c|c|c|c|c|c|}
\hline Biohorizon & $\begin{array}{l}\text { Age } \\
\text { (Ma) }\end{array}$ & $\begin{array}{c}\text { Hole } 812 \mathrm{~A} \\
\text { Depth (mbsf) }\end{array}$ & $\begin{array}{l}\text { Hole } 812 \mathrm{C} \\
\text { Depth (mbsf) }\end{array}$ & $\begin{array}{c}\text { Hole } 813 \mathrm{~A} \\
\text { Depth (mbsf) }\end{array}$ & $\begin{array}{c}\text { Hole } 814 \mathrm{~A} \\
\text { Depth (mbsf) }\end{array}$ & $\begin{array}{c}\text { Hole 815A } \\
\text { Depth (mbsf) }\end{array}$ & $\begin{array}{c}\text { Hole } 816 \mathrm{~A} \\
\text { Depth (mbsf) }\end{array}$ & $\begin{array}{c}\text { Hole } 817 \mathrm{~A} \\
\text { Depth (mbsf) }\end{array}$ & $\begin{array}{c}\text { Hole 818B } \\
\text { Depth (mbsf) }\end{array}$ \\
\hline Emiliania huxlevi LO & 0.275 & 2.67 & & 2.63 & 4.12 & 1.1 & 0.37 & 25.07 & 9.53 \\
\hline Pseudoemiliania lacumosa HO & 0.465 & 7.52 & & 9.83 & 10.01 & 4.1 & 1.87 & 28.07 & 14.03 \\
\hline small Gephyrocapsa Acme top & 0.93 & 17.02 & & 23.82 & 21.01 & 13.9 & 4.87 & 45.56 & 53.51 \\
\hline small Gephymocapsa Acme bottom & 1.1 & & & & & 17.4 & & 51.56 & \\
\hline Helicosphaera sellii $\mathrm{HO}$ & 1.27 & & & & & 18.9 & & & \\
\hline Calcidiscus macintrrei $\mathrm{HO}$ & 1.48 & 23.9 & $23.27-24.77$ & 33.32 & 29.01 & 20.4 & 9.62 & 63.06 & 80.51 \\
\hline Gephyrocapsa nceanica s.I. LO & 1.59 & & & & & & & 72.56 & \\
\hline Discoaster bromeri $\mathrm{HO}$ & 1.88 & & 25.9 & 39.81 & 33.51 & 24.9 & 12.62 & 72.56 & 84.58 \\
\hline Discouster pentaradiatus HO & 2.29 & $25.96-27.90$ & $26.85-29.0$ & 52.31 & 41.51 & 31.4 & 15.18 & 72.56 & 84.58 \\
\hline Discoaster surculus HO & 2.42 & & & 53.81 & 44.51 & 36.4 & 17.62 & 72.56 & 106.01 \\
\hline Discoaster asymmetricus $\mathrm{HO}$ & 2.42 & & & & & & & & \\
\hline Discoaster tamalis HO & 2.6 & & & 57.31 & 52.51 & 44.72 & 19.12 & 75.56 & 177.02 \\
\hline Discoaster variabilis HO & 2.9 & & & & & & & & \\
\hline Sphenolithus abies HO & 3.45 & $29.26-35.40$ & & 79.31 & 66.83 & $|16.9|$ & 43.5 & 155.7 & 246.08 \\
\hline Reticulofenestra pseudoumbilicus $\mathrm{HO}$ & 3.51 & & 53.61 & 80.81 & & 158.9 & 76.11 & 155.7 & 259.51 \\
\hline Pseudoemiliania lacunosa LO & 4.21 & & & & & 309.46 & & 161.06 & \\
\hline Amaumlithus tricomiculatus $\mathrm{HO}$ & 4.24 & & & & & 309.46 & & 161.06 & \\
\hline Ceratolithus armatus HO & 4.6 & & & & & 326.35 & & & \\
\hline Amaurolithus tricomiculanus LO & 4.7 & & & & & 331.61 & & 176.7 & \\
\hline Ceratolithus rugosus LO & 4.72 & & & & & & & 176.7 & \\
\hline Ceratolithus armatus LO & 5.06 & & & & & 351 & & & \\
\hline Discoaster quinqueramus HO & 5.26 & & 85.61 & 99.81 & 76 & 354 & & 180.07 & \\
\hline Amaurolithus amplificus HO & 5.6 & & & & & 378.54 & & 186.07 & \\
\hline Discoaster berggrenii HO & 5.8 & & & & & 377.04 & & 189.57 & \\
\hline Amaurolithus amplificus LO & 5.9 & & & & & 387.3 & & 195.57 & \\
\hline Amaurolithus primus/A. delicatus $\mathrm{LO}$ & 6.74 & & & & & 409.36 & & 200.56 & \\
\hline
\end{tabular}

"Core loss. $\mathrm{LO}=$ lowest occurrence: $\mathrm{HO}=$ highest occurrence.

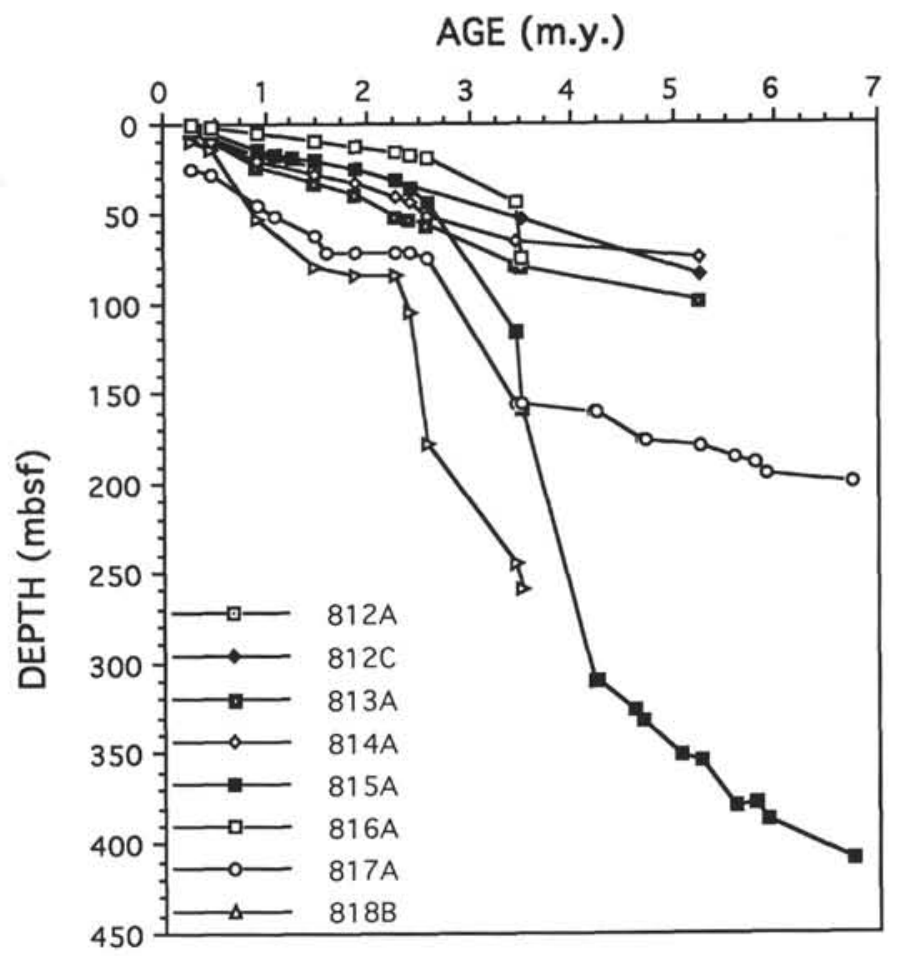

Figure 12. Plots of age vs, depth for all sites from the southern transect of Townsville Trough. 